Sādhanā Vol. 38, Part 6, December 2013, pp. 1135-1171. (C) Indian Academy of Sciences

\title{
Mathematical modelling of dropwise condensation on textured surfaces
}

\author{
BASANT SINGH SIKARWAR, SAMEER KHANDEKAR* and \\ K MURALIDHAR
}

Department of Mechanical Engineering, Indian Institute of Technology Kanpur, Kanpur 208 016, India

e-mail: samkhan@iitk.ac.in

MS received 4 March 2012; revised 23 July 2012; accepted 26 July 2012

\begin{abstract}
Vapor-to-liquid phase change in the form of discrete drops on or underneath a substrate is called dropwise condensation. The process is hierarchical in the sense that it occurs over a wide range of length and timescales. As the associated heat transfer coefficient is much higher than the film and mixed mode of condensation, it is of considerable interest in applications. The present study is focused on mathematical modelling of dropwise condensation process at multiple scales. The model includes formation of drops at the atomistic scale, droplet growth, coalescence, instability, slide off and fall-off, followed by fresh nucleation of liquid droplets. The model shows that the largest stable cluster size in the atomic model matches the minimum drop radius estimated from thermodynamic considerations. The minimum drop radius is insensitive to surface texturing and does not provide controllability at larger length and timescales. A closer examination of droplet distribution over the substrate reveals that small drops are locations of high heat transfer rates, which diminishes with increasing drop radius. The largest drop diameter depends on its stability and hence, the interfacial forces at phase boundaries. Therefore, drop instability controls the heat transfer coefficient in dropwise condensation. Enhancement of heat transfer necessitates that these drops grow with time, become unstable and be swept away as quickly as possible. Enhancement may be achieved either by (i) inclining the substrate or (ii) by creating an interfacial force at the three-phase contact line by a wettability gradient over the horizontal substrate, inducing drop motion. Wall heat transfer and shear stress under moving drops have been determined using a CFD model. A simple model of coalescence has been adopted in this work. Simulation studies on the effect of fluid properties, surface inclination and its wettability condition on drop size distribution, cycle time, heat transfer coefficient, and wall shear stress are comprehensively discussed in the present article.
\end{abstract}

Keywords. Dropwise condensation; hierarchical modelling; wettability; horizontal and inclined surfaces; pendant drops; heat transfer.

*For correspondence 


\section{Introduction}

Heterogeneous condensation of vapor on or underneath a surface is one of the most efficient ways of heat transfer in engineering systems, the resulting heat transfer coefficient being orders of magnitude larger than single-phase convective paradigms. This phase-change process may result, under quasi-steady-state conditions, in either, (i) the formation of a droplet ensemble of the condensing fluid, (ii) the formation of a continuous film on the cold substrate or, (iii) there can be a mixed mode too, having fuzzy overlapping characteristics of drops and a liquid film. Phasechange in the form of discrete drops on or underneath a substrate is called heterogeneous dropwise condensation and the associated heat transfer coefficient is at least one order higher than the film and mixed mode of condensation (Carey 1992; Rose 2002). The formation of discrete drops can be promoted by treating the surface in such a way that it becomes lyophobic. Dropwise condensation is necessarily a spatio-temporal cyclic process with a chain of intermediate processes such as initial nucleation, growth of drops by direct condensation, coalescence, drop instability and movement followed by fresh nucleation, as detailed in figure 1 (Sikarwar et al 2011a).

The growth processes occurring in dropwise condensation cover a wide spectrum of length and timescales (Tien et al 1997). After hot vapor atoms/molecules strike a cold virgin substrate, at the atomic scale, some vapor molecules called adatoms get bound to the surface and undergo surface diffusion; eventually a group of adatoms leads to stable clusters. Stable clusters growing together may form a molecular monolayer of condensate on the substrate. At this scale, there are two prevalent theories of drop formation as schematically depicted in figure 2 . In the first, it is postulated that the condensation initially occurs in a film-wise manner, forming an extremely thin film on the solid surface. As condensation continues in time, the film ruptures due to intrinsic interfacial instabilities forming distinct droplets via spinodal dewetting. The second theory

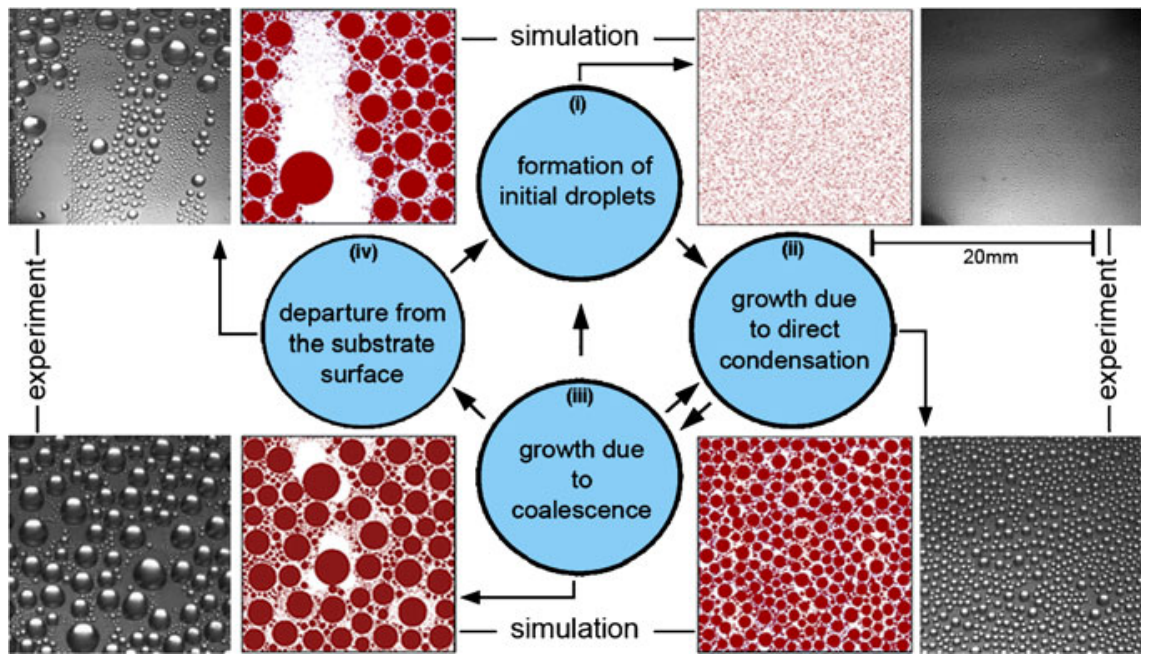

Figure 1. Cycle of individual processes at various time instants in condensation of vapor over an inclined substrate; inter-relationship shown among the distinct processes are: (i) nucleation and growth of drops at each nucleation site by direct vapor condensation; (ii) joint growth by direct condensation and coalescence; (iii) growth of large drops mainly by coalescence and smaller drops by direct condensation; and (iv) drop instability and condensate removal from the substrate. Fresh nucleation (step i) is initiated at the newly exposed sites. 
(a) atoms, adatoms and unstable cluster

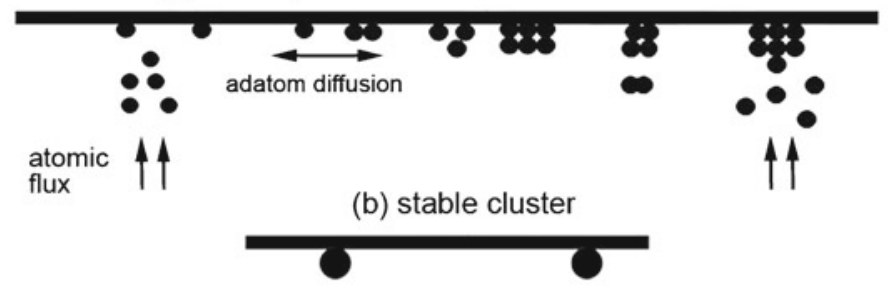

(c) stable cluster growth

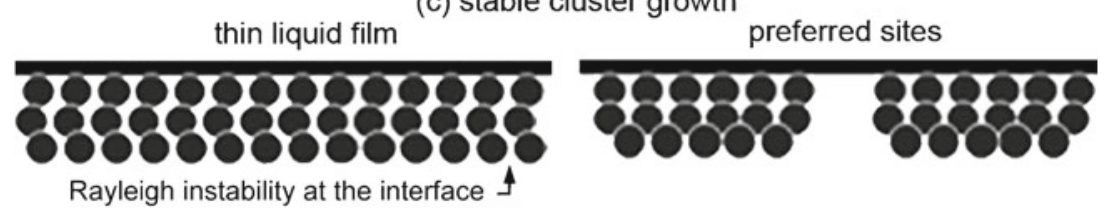

(d) drop formation

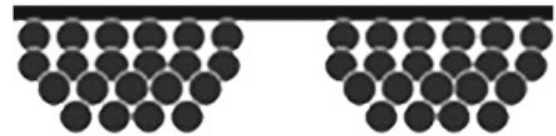

spinodal dewetting (intrinsic)

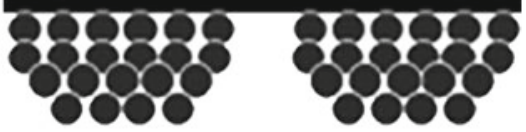

nucleation dewetting (extrinsic)

Figure 2. Mechanism of liquid drop formation underneath a cold substrate. From atomic level to molecular stable clusters, individual vapor molecules come closer, a system of adatoms form, a group of adatoms leads to a cluster. Many growing clusters together may form a molecular monolayer of liquid. At this stage there are at least two possibilities: droplet formation (dewetting) and film formation. This liquid film ruptures and form droplets (adapted from Sikarwar 2012).

is based on the premise that drop formation is a heterogeneous nucleation process. Here, a stable cluster gets located at specific nucleation sites over the substrate, such as pits and grooves, grow in the continuum domain, while the portion of the surface between growing drops essentially remains dry. Small droplets are formed by direct condensation via nucleation dewetting at locations with local minima of the free surface energy.

In macroscopic modelling of dropwise condensation, nucleation sites are distributed over an area using appropriate stochastic distribution functions. Thereafter, such randomized site locations remain fixed for a given simulation. Studies show that single drops of the size of the largest molecular cluster, determined from atomistic considerations, are equal in diameter to the thermodynamically minimum stable drop radius. Such drops appear at preferred nucleation sites over the substrate. The drop will subsequently grow at the continuum scale, as discussed below.

At the continuum scale, two mechanisms of heat transfer through the drop have been reported in the literature (Rose et al 1999; Sikarwar et al 2011b). In the first, vapor condenses between the droplets, in the droplet-free areas. The condensate is transported to the droplets by surface diffusion within an adsorbed liquid thin film, wherein the conduction resistance to heat transfers in the boundary-layer can be neglected, leading to very large heat transfer coefficients. According to this latter model, heat transfer takes place both, within an extremely thin liquid film (in-between the drops), as well as through the droplets, though the major part of heat transfer takes place through the former. Larger droplets mainly act as collection sites for the liquid. The second model is based on the premise that droplets are formed at individual nucleation sites, 
while the area between the droplets is inactive with respect to condensation. Heat transfer occurs only through the droplets and is limited by their conduction resistance, which decreases with increasing drop size. According to this model, drops grow by direct condensation and later by both direct condensation and coalescence with neighbouring droplets. Most experimental results support the second mechanism of heat transfer during dropwise condensation.

Dropwise condensation is intrinsically unsteady because drops grow and reach a critical size, beyond which they slide-off or fall-off from the substrate. The preference of one critical mode over the other depends on the substrate orientation. On an inclined substrate, the weight component of the drop parallel to the surface exceeds the retention force between the droplet and the substrate at the three-phase contact line. The drop then begins to slide along the surface and wipes other drops along its path, leading to new clean exposed surface areas. Fresh droplets renucleate at nucleation sites thus becoming available on the newly exposed surface for growth. This process of continuous wiping of the substrate and the formation of new small drops is the reason for the ensuing large heat transfer coefficient. The removal of condensate by drop instability is an essential step for heat transfer enhancement in dropwise condensation (Leipertz 2010; Kim \& Kim 2011). The consequence of drop instability could be a rolling motion as well, a possibility that is expected in super hydrophobic surfaces but is not considered here.

Advances in nanotechnology, physical and chemical texturing and thin film coatings generate various possibilities for passive mobilization of drops on a substrate (Wang et al 2003; Subramanian et al 2005; Qiang et al 2006; Pratap et al 2008). A simple approach is to incline the surface with respect to the horizontal. The others are: (a) apply a large temperature gradient on the hydrophobic substrate so that Marangoni convection is initiated, (b) create wettability gradient on the substrate by a suitable chemical treatment. Various authors report that the second approach is realizable by depositing organic long chain monolayers over the substrate (Daniel et al 2001; Moumen et al 2006). The advantage of this technique is that the micro-drops can move on or underneath a horizontal substrate and substrate inclination is not required. This approach has potential applications in a zero-gravity environment. The sweeping action of drops on a surface with a wettability gradient can, further augment heat transfer coefficient (Lan et al 2009).

\subsection{Physical and chemical texturing}

Parameters, including the average contact angle, contact angle hysteresis and the nucleation site density of chemically textured surfaces can be quite different from the physically textured counterparts. These parameters are an input to the condensation model reported in the present study. When these parameters are available for any given textured surface, the model can be used to study dropwise condensation.

Physically textured surfaces are unique in many different ways for the following reasons. For a single drop of liquid sitting over a physically patterned surface, multiple droplet configurations are possible, making the determination and interpretation of the apparent contact angle a challenge. For example, the static drop could exhibit wetting transitions between Cassie state or the Wenzel state (Berthier 2008; Miljkovic et al 2012); such configurations of droplets over physically textured surfaces can be seen, for example, in Berthier (2008), Ma et al (2012) and Rykaczewski (2012). Hence, identifying a unique contact angle could be challenging. However, for a continuous cyclic process of dropwise condensation on randomized hydrophobic textured surfaces, encountering an ensemble of drops of various sizes, it may be argued that the bulk behaviour of physically and chemically textured surfaces could be comparable, except for some differences in apparent dynamic contact angles and the mobility of the three-phase contact line. 
These differences should be small when the drop size is large in comparison to the characteristic scale of surface roughness. As the drop size at criticality is of the order of a few mm (for the fluids studied), the drop is much larger than the surface features and therefore, the proposed condensation model is expected to be uniformly valid for physically as well as chemically textured surfaces, provided applicable parameters are available as input to the model, as indicated earlier.

\subsection{Mathematical modelling}

Although, dropwise condensation on engineered surfaces is an attractive technique for enhancing heat transfer, modelling this cyclic process is complex and depends on complex interactions of surface, body and pressure forces. A unified model that can predict major spatio-temporal transport parameters, for a given condensing medium and substrate characteristics, has not been reported earlier and is the focus of the present article. With the drop level information determined, one obtains the local/average heat transfer coefficients. The resulting wall shear stresses provide vital information for predicting substrate leaching rates due to continuous motion of drops over it; this limits the long term sustainability of the process in engineering systems.

A comprehensive mathematical model of dropwise condensation over inclined substrates is discussed in the following sections. The model is simulated as a computer code in $\mathrm{C}++$. Wherever possible, the model has been validated against experimental and analytical data reported in the literature. Transport parameter computations (e.g., spatial patterns of condensation, heat transfer rates and wall shear stresses) for various contact angles, initial nucleation sites, hysteresis, wettability gradient and substrate inclination angle, are reported.

The paper is organized in the following sequence: description of mathematical modelling of dropwise condensation, starting from nucleation, direct condensation growth, coalescence, and instability, calculation of heat transfer and shear stress in moving drops, results and discussion, and conclusions.

\section{Modelling of dropwise condensation}

A framework that explains hierarchical modelling of dropwise condensation, in terms of the processes involved, is depicted in figure 3. The atomistic model, which relies on population balance, is the starting point for determining the size of the smallest stable drop. The subsequent steps that follow are growth by direct condensation, coalescence, instability, drop motion, and computation of heat transfer and wall shear for sliding drops. The model as a whole yields the instantaneous drop size distribution over inclined surfaces.

\subsection{Atomistic modelling of dropwise condensation}

Formation of drops during condensation commences with the impingement of vapor atoms on a cold substrate, kept at a temperature below saturation (Amar et al 1999; Venables 2000; Tian et al 2004). Atoms approaching the cold wall have higher energies than departing atoms and hence transfer energy to the wall. The energy exchange between incoming and departing atoms of different energies (temperatures) provides a sufficient condition for molecular clustering. Clustering may begin in the vapor phase itself, close to the cold substrate wall. A kinetic balance model at the atomic level, which has been reported by various researchers (Brune 1998; Venables \& Brune 2002; Oura et al 2003; Shi et al 2005), was used by authors (Sikarwar et al 


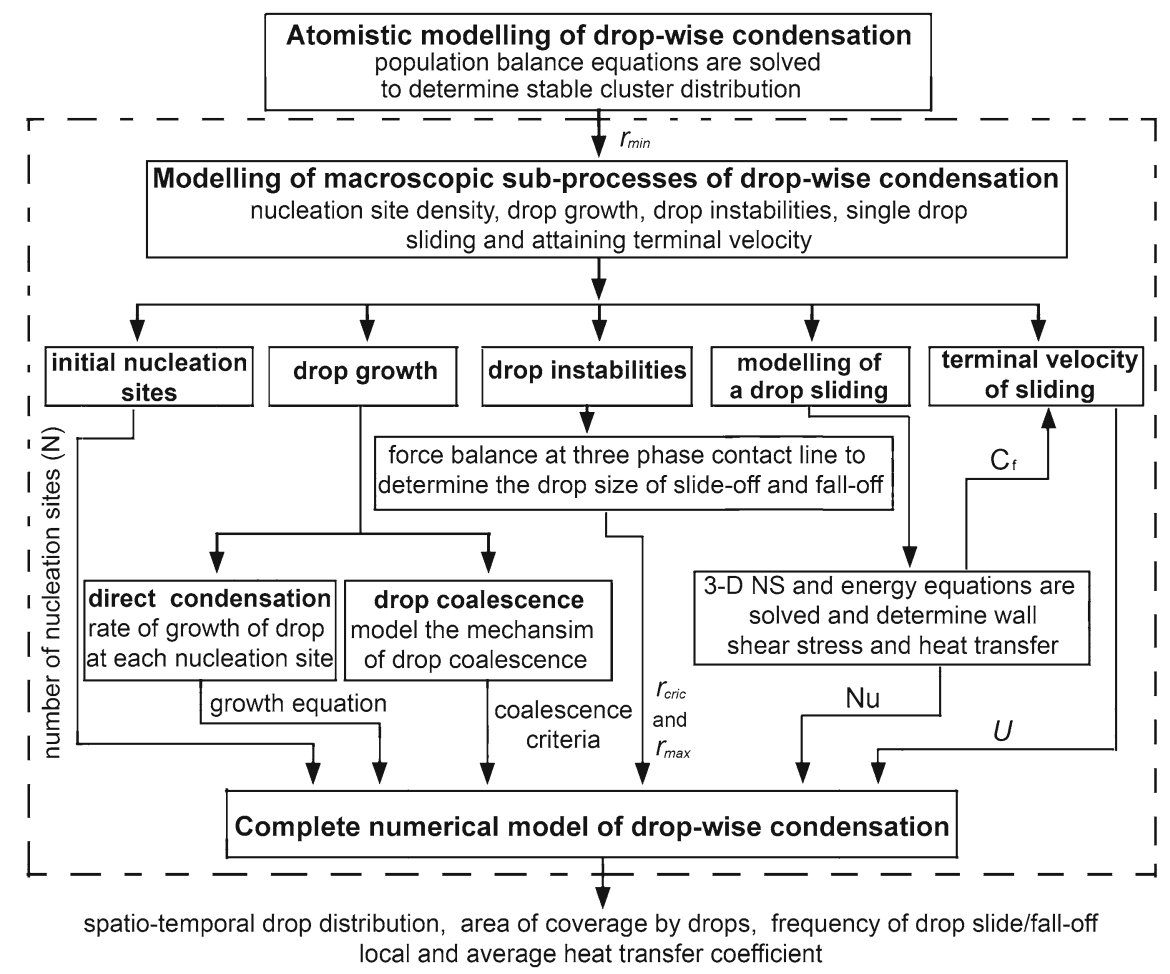

Figure 3. Block diagram of multi-scale modelling of dropwise condensation. The atomistic scale model provides the minimum size of the drop. Modelling of stability of single drops gives the maximum size of drop at slide-off or fall-off. In addition, subsequent modelling steps involve drop coalescence and calculation of wall shear and heat transfer coefficient.

2012) to predict the size of the smallest stable cluster during condensation of saturated vapor on a sub-cooled substrate. The summary of which is the following: A population balance model is described in terms of rate equations to obtain the number density distribution of clusters. The residence time is taken to be large enough so that sufficient time is available for all the adatoms existing in vapor-phase to lose their latent heat and get condensed. The simulation assumes clusters of a given size to be formed from clusters of smaller sizes but not by the disintegration of larger clusters. The largest stable cluster size in the number density distribution is taken to be representative of the minimum drop radius formed in a dropwise condensation process. A numerical confirmation of this result against predictions based on a thermodynamic model has been obtained. Results show that the number density distribution is sensitive to the surface diffusion coefficient and the rate of vapor flux impinging on the substrate. The minimum drop radius increases with the diffusion coefficient and the impinging vapor flux but the dependence is weak. The minimum drop radius predicted from thermodynamic considerations matches the prediction of the cluster model, though the former does not take into account the effect of the surface properties on the nucleation phenomena. For a chemically passive surface, the diffusion coefficient and the residence time are dependent on the surface texture via the coefficient of friction. Thus, physical texturing provides a means of changing, within limits, the minimum drop radius. The study reveals that surface texturing at the scale of the minimum drop radius does not 
provide controllability of macro-scale dropwise condensation at large timescales when a dynamic steady-state is reached.

\subsection{Modelling of macroscopic sub-processes of dropwise condensation}

Dropwise condensation is a consequence of the time-dependant sub-processes associated with the formation of the drops, growth by direct condensation and coalescence, sliding motion, fall-off and then by renucleation on or underneath the substrate. It is a complex phenomenon involving several parameters which are intricately linked. A mathematical model of these subprocesses is required to entirely describe the dropwise condensation process, as described hereunder.

2.2a Nucleation site density: The size of initial, thermodynamically determined, drops are of the order of magnitude of a few nanometers for fluids encountered in heat transfer. Therefore, from an engineering standpoint, it is difficult to experimentally capture the initial nucleation phenomenon on a surface freshly exposed to vapor. Nucleation site density is itself influenced by the thermophysical properties of the condensing fluid, physico-chemical properties of the substrate, degree of subcooling and the substrate morphology. Thus, it is also difficult to determine the nucleation site density on a substrate, either from theory or experiments. Leach et al (2006) reported initial site densities close to $10^{6} \mathrm{~cm}^{-2}$ for temperature differences in the range of 50 to $100^{\circ} \mathrm{C}$. They suggested that for condensation of water at $30^{\circ} \mathrm{C}$, the initial nucleation site density is in the range of $10^{4}$ to $10^{5} \mathrm{~cm}^{-2}$, and gradually increases to $10^{6} \mathrm{~cm}^{-2}$ before the first coalescence. Rose (2002), also indicated that the parameter $N$ in the range of $10^{5}-10^{6}$ is close to experimental data of dropwise condensation underneath a chemically textured substrate. Earlier, a theoretical expression for nucleation site density over an untreated surface was given by Rose (1976) as

$$
N=\frac{0.037}{r_{\min }^{2}} \mathrm{~cm}^{-2} \text { where, } \quad r_{\min }=\frac{2 \sigma \cdot v_{l} \cdot T_{w}}{h_{l v}\left[T_{\text {sat }}-T_{w}\right]}
$$

Here, $N$ is the number of sites on the substrate per unit area where the initial drops, identifiable as liquid, are formed. Zhao \& Beysens (1996) observed no significant connection between the initial nucleation site density and the wettability of the condensing fluid. Later, Mu et al (2008) found that the nucleation density varies with surface topography, the rougher substrate resulting in a higher nucleation density. Based on the work of Rose (1976) and Mu et al (2008), one can conclude that the nucleation density is influenced not only by the degree the surface topography but also by sub-cooling. In dropwise condensation, the substrate morphology could be changed by chemical and physical texturing. Hence, nucleation density might be influenced by these two factors, i.e., changes in surface energy induced by a chemical species (chemical texturing) and varying roughness morphology of the substrate (physical texturing). The modified expression for the nucleation density of a textured substrate can be expressed as

$$
N_{f}=f N
$$

Here, $N_{f}$ is the nucleation site density of the textured substrate, $f$ is the degree of roughness and $N$ is initial nucleation density of a smooth surface, as calculated by Equation (1), Rose (1976). For a general textured substrate - physical or chemical, factor $f$ needs to be established and is a contemporary topic of research. 
As indicated above, typically there are two broad methods by which nucleation site density can be estimated, (i) either an indicative value can be directly used as a direct input to the model or, alternatively, (ii) a value of degree of roughness, $f$, of substrate can be used in conjunction with Equation (1) and (2) to obtain, $N$. The approach adopted in the present work is to examine the sensitivity of a macroscopic quantity, such as average heat transfer rate, to the nucleation site density, over a wide range of values. Based on the indicative data, it is believed that an input value of $N=10^{6} \mathrm{~cm}^{-2}$ is a good approximation for initial calculations. Subsequently, the parametric effect of nucleation site density and degree of subcooling on dropwise condensation heat transfer has been independently studied.

From the view point of the current model, the nucleation sites are randomly distributed over the substrate area by a random seed generator function in $\mathrm{C}++$ that returns a matrix containing pseudo random numbers with a uniform probability density function in the range $[0,1]$. The distribution of sites over the area proceeds column-wise till all the sites are occupied (Sikarwar et al 2011d). Once this distribution is done, it remains fixed for a given simulation. Unless otherwise stated, we have used nucleation site density of $10^{6} / \mathrm{cm}^{2}$ in all simulations reported here. According to the model developed in the present study, a high nucleation site density leads to a reduction in the size of drops that experience first coalescence. Early coalescence creates space for fresh nucleation, increasing the population of small diameter drops. Correspondingly, the heat transfer coefficients are also higher.

2.2b Drop growth by direct condensation: The rate of condensation on the free surface of a drop depends on its ability to transfer the latent heat release to the cooler substrate through its own volume, figure 4. For the present discussion, the drop is considered to be a part of a sphere with contact angle $\theta_{\text {avg. }}$. For the horizontal substrate without any wettability gradient,

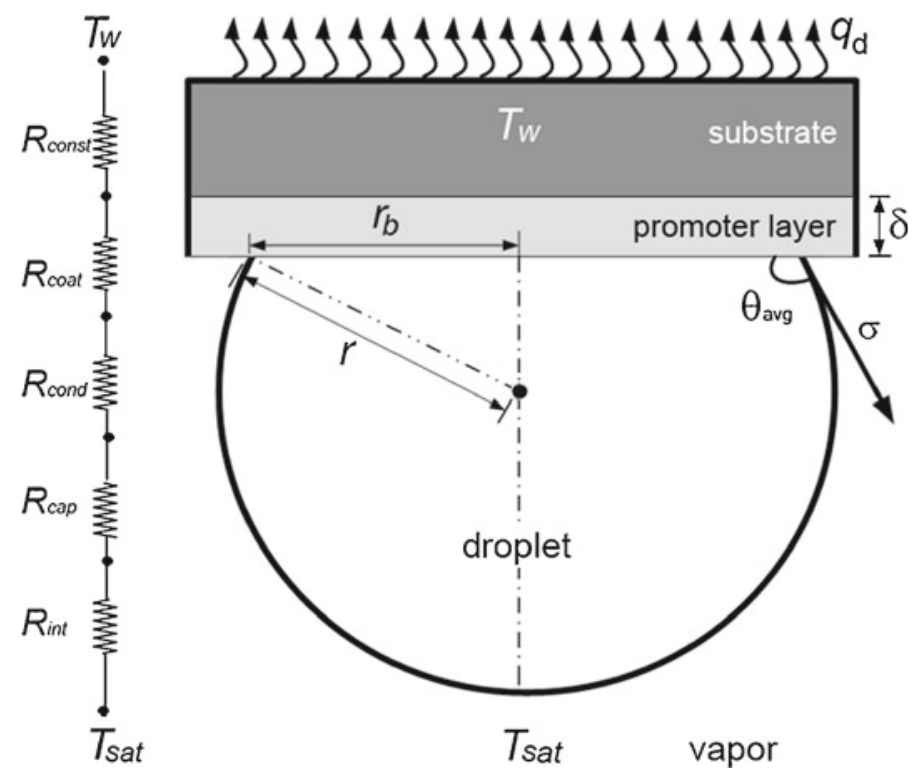

Figure 4. Schematic diagram of a pendant drop underneath a horizontal substrate with thermal resistances in the droplet growth equation, shown. The promoter layer has a thickness $\delta, T_{s a t}$ is the vapor saturation temperature, and $T_{w}$ is the wall temperature. 
the average apparent contact angle $\left(\theta_{\text {avg }}\right)$ is equal to apparent contact angle $(\theta)$. However, for (i) horizontal substrate with a wettability gradient and, (ii) an inclined substrate without any wettability gradient, $\theta_{\text {avg }}$ assumes different values, the estimation of which is discussed in Sections 2.2c and 2.2d.

The thermal resistances, relevant to heat transfer through an isolated drop, are discussed below.

Conduction resistance: The temperature difference associated with conduction heat transfer through the drop is determined as

$$
\Delta T_{\text {cond }}=\frac{q_{d} r\left(1-\cos \theta_{a v g}\right)}{4 \pi r^{2} k_{l}} .
$$

Film resistance: Temperature difference due to the film resistance at the vapor - liquid interface is

$$
\Delta T_{i n t}=\frac{q_{d}}{2 \pi r^{2} h_{i n t}\left(1-\cos \theta_{a v g}\right)} .
$$

Curvature resistance: It includes the loss of temperature potential due to multi-dimensional heat conduction arising from the curvature of the drop free surface and is given by (Carey 1992)

$$
\Delta T_{\text {curv }}=\left(\frac{2 \sigma}{r}\right)\left(\frac{v_{l} T_{w}}{h_{l v}}\right)=\frac{\left(T_{s a t}-T_{w}\right) r_{\min }}{r} .
$$

Coating resistance: The temperature drop due to the resistance offered by the coating material, if applied on the substrate, is given by

$$
\Delta T_{\text {coat }}=\frac{q_{d} \delta}{k_{\text {coat }} \pi r^{2}\left(1-\cos ^{2} \theta_{a v g}\right)} .
$$

Constriction resistance: It measures the effect of surface thermal resistance on dropwise condensation. This is caused due to non-uniform heat flux distribution over the condensing surface. Although, there is continuing controversy on whether thermal conductivity of the condensing surface has a significant role on the rate of droplet growth, it is included here, following the work of Mikic (1969). ${ }^{1}$ Hence,

$$
R_{\text {const }}=\frac{8}{3 \pi k_{w}} \sum_{r_{\min }}^{r_{\max }} \frac{n_{r} r^{3}}{[1-f(r)]},
$$

where, $n_{r}$ is nucleation site density for a drop of radius $r$ and $f(r)$ is the fraction of the surface area covered by drops of radius $r$. The associate temperature penalty is,

$$
\Delta T_{\text {const }}=\frac{q_{d}}{R_{\text {const }}}
$$

\footnotetext{
${ }^{1}$ Although incorporated in the computer code, results discussed here do not include the effect of constriction resistance.
} 
The total temperature drop will balance the total available sub-cooling and thus,

$$
\Delta T_{t}=\Delta T_{\text {cond }}+\Delta T_{\text {int }}+\Delta T_{\text {curv }}+\Delta T_{\text {coat }}+\Delta T_{\text {const }}=\left(T_{\text {sat }}-T_{w}\right)
$$

The net heat transfer through a single drop is derived as

$$
q_{d}=\left(\pi r^{2} \rho_{l} h_{l v}\right) \cdot\left(2-3 \cos \theta+\cos ^{3} \theta\right) \cdot(d r / d t) .
$$

From the above set of equations, the rate of growth of individual drops follows the equation:

$$
\begin{aligned}
\frac{d r}{d t}= & \left(\frac{4\left(T_{\text {sat }}-T_{w}\right)}{\rho h_{l v}}\right) \\
& \times\left[\frac{\left(1-\frac{r}{r_{\text {min }}}\right)}{\left(\frac{2}{h_{\text {int }}}+\frac{r\left(1-\cos \theta_{\text {avg }}\right)}{k_{l}}+\frac{\delta}{k_{\text {coat }}\left(1+\cos \theta_{\text {avg }}\right)}+R_{\text {const }} 4 \pi r^{2}\left(1-\cos \theta_{\text {avg }}\right)\right)}\right] \\
& \times\left[\frac{\left(1-\cos \theta_{\text {avg }}\right)}{\left(2-3 \cos \theta_{\text {avg }}+\cos ^{3} \theta_{\text {avg }}\right)}\right] .
\end{aligned}
$$

2.2c Drop coalescence: Literature emphasizing the relationship between coalescence of sessile and pendant drops and the role of coalescence in heat transfer enhancement is scarce. Therefore, the authors of the present work have performed an experiment involving the coalescence of two water drops in the pendant mode. The layout of the experiment set-up is shown in figure 5a. Figure 5b shows two drops coalescing on a hydrophobic surface. Images were recorded by a high speed camera (at $7500 \mathrm{fps}$ ) underneath a Teflon substrate. The time and length scales of coalescence for various sizes of the drops were observed Sikarwar et al (2012). A typical time sequence of snapshots, when two drops of differing diameters merge, is shown in figure $5 \mathrm{c}$. The entire time period of the dropwise condensation cycle, from a virgin surface to the first fall-off or a slide-off, is typically of the order of several minutes; in contrast, coalescence happens within a time period of 5-60 ms. This timescale, though very small, depends on the size of the individual drops and other thermo-physical properties such as viscosity, surface tension, contact angles, and surface texture conditions at the operating temperature.

Based on these preliminary observations, it is to be noted that:

(i) When two drops of different sizes coalesce, the flow is into the larger drop as it is a region of lower pressure. Pressure differences arise from drop curvature, and hence surface tension. A number of new nucleation sites also get exposed on the freshly uncovered condensing surface.

(ii) When two large drops of nearly equal volume coalesce, they form a single drop and depending on their initial sizes, the newly formed drop may exceed the critical size, resulting in its sliding motion or a fall-off. A number of nucleation sites are once again exposed.

(iii) The process of coalescence itself induces fluid motion and enhanced convection. In addition, drop coalescence also momentarily reduces the pressure acting on the surface, creating time-dependent pressure fluctuations. These factors will invariably enhance the local heat transfer coefficient. This observation needs further numerical and experimental exploration. 
(a)

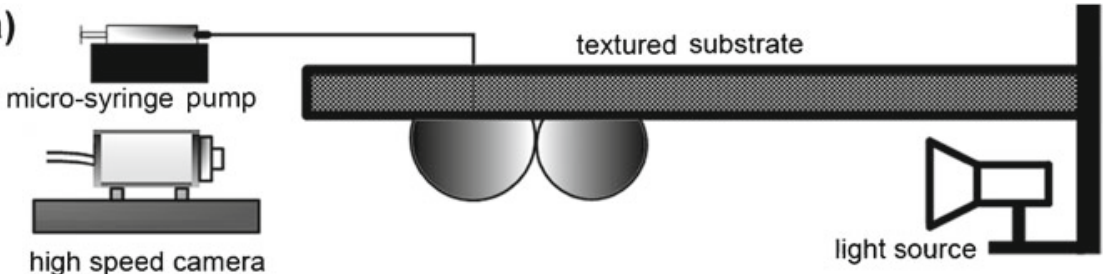

(b)

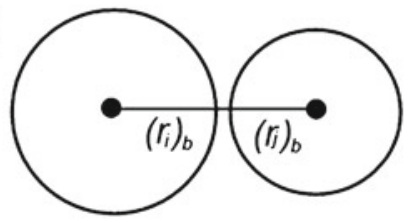

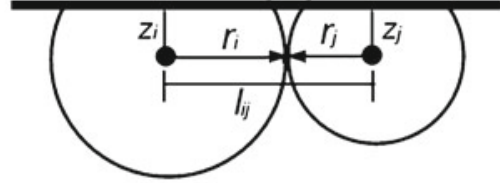

two drops before coalescence

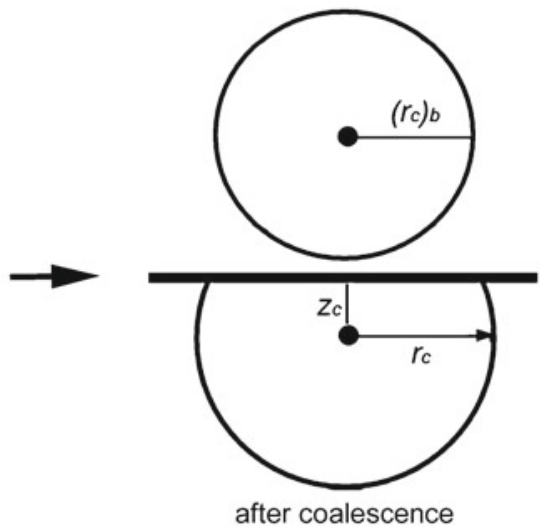

after coalescence

(c) Two water pendant droplets coalescing at room temperature drop A drop B

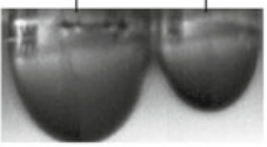

$\mathrm{t}=0.1 \mathrm{~ms}$

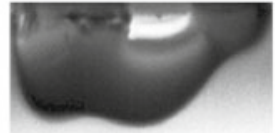

2.10

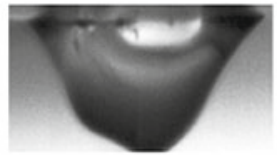

10.56

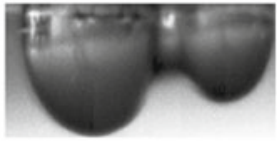

0.2

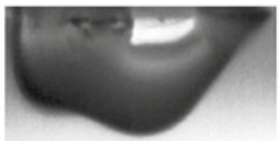

3.01

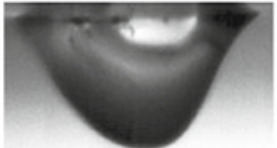

12.10

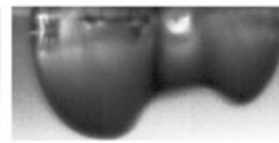

0.35

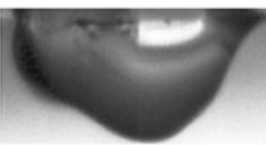

4.00

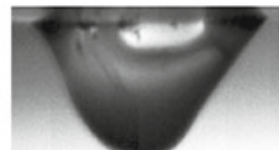

14.01

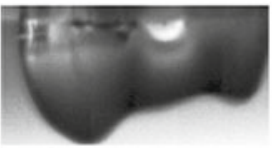

0.45

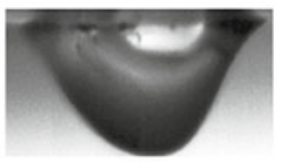

9.62

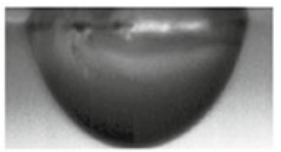

$\mathrm{t}=60.00 \mathrm{~ms}$

radius of $\operatorname{drop} A=2 \mathrm{~mm}$; radius of $\operatorname{drop} B=1 \mathrm{~mm}$

Contact angle on substrate $=98^{\circ}$

Figure 5. (a) Schematic diagram of the setup used for visualizing coalescence of pendant drops; (b) coalescence of two drops and criteria of coalescence with terminology; and (c) image captured by a high speed camera during the coalescence of water drops underneath Teflon sheet. 
In the above discussion, the substrate is taken to be at a constant temperature. Condensation affects the wall heat flux transferred from the vapor to the wall (Bansal et al 2009). In practice, wall temperature fluctuations are likely, particularly if the thermal inertia of the substrate matches the timescale of drop instability.

On the basis of the above observations, drop coalescence is modelled in dropwise condensation as follows. The distance between two nucleation sites, $i$ and $j$, on the substrate (figure $5 \mathrm{~b}$ ) is calculated as

$$
l_{i j}=\sqrt{\left(x_{i}-x_{j}\right)^{2}+\left(y_{i}-y_{j}\right)^{2}+\left(z_{i}-z_{j}\right)^{2}} .
$$

Check for coalescence; the coalescence criterion can be stated $\mathrm{as}^{2}$

$$
l_{i j}-\left[r_{i}+r_{j}\right]<10^{-3} \text {. }
$$

If coalescence criterion is met, a drop of equivalent volume on the mass averaged center of the original coalescing droplets is introduced. The time for coalescence is taken to be much smaller than the other timescales of the condensation process. Hence, as soon as two droplets contact each other (or three droplets, or, very rarely, four contact each other simultaneously), they are substituted with an equivalent single drop with equal total volume, located at the weighted center of mass of the individual coalescing drops. Figure 5b shows two drops, $i$ and $j$ in a coalescence process forming drop $c$. The radius of $\operatorname{drop} c$ is

$$
r_{c}=\left[\frac{3\left(V_{i}+V_{j}\right)}{\pi\left(2-3 \cos \theta_{\text {avg }}+\cos ^{3} \theta_{\text {avg }}\right)}\right]^{1 / 3} .
$$

The base radius of the drop formed after coalescence is

$$
\left(r_{c}\right)_{b}=r_{c} \sin \theta_{\text {avg }}
$$

Here, $r_{c}$ is the radius of the new drop formed after coalescence and $V_{i}$ and $V_{j}$ are volumes of drops $i$ and $j$, respectively. The average contact angle for a horizontal surface with unidirectional wettability gradient varies from drop to drop. For $i^{\text {th }}$ drop, it is given as

$$
\left(\theta_{\text {avg }}\right)_{i}=\left[\left(\theta_{\max }\right)_{i}+\left(\theta_{\min }\right)_{i}\right] / 2 \text {. }
$$

For an inclined substrate, the average contact angle is given as

$$
\theta_{\text {avg }}=\left(\theta_{r c d}+\theta_{a d v}\right) / 2 \text {. }
$$

2.2d Drop instability: During condensation, drops grow first by direct deposition of vapor and then by coalescence. Continuously, the weight of the drop increases and can be a destabilizing influence. Hence, an imbalance of forces at the three-phase contact line leads to instability. For definiteness, a pendant drop underneath a flat horizontal substrate, figure $6 \mathrm{a}-\mathrm{i}$, is considered. It is

\footnotetext{
${ }^{2}$ For a horizontal surface with a wettability gradient and an inclined surface, the effective radius of the drop is obtained from the spherical cap approximation, as shown in figure 6.
} 
(a)

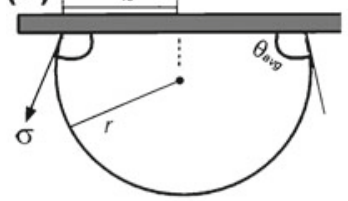

pendant drop underneath horizontal substrate

(b)

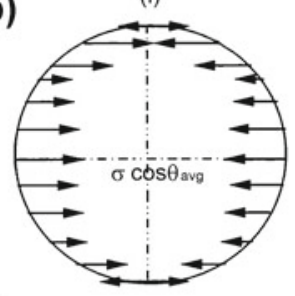

(c)

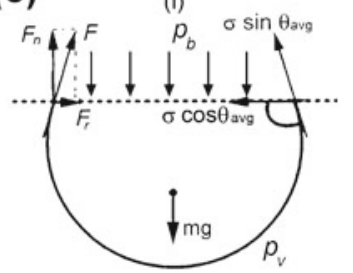

(ii)

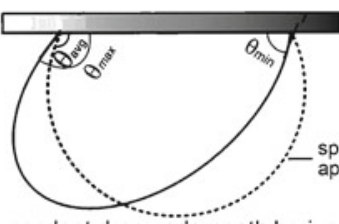

pendant drop underneath horizontal with wettability gradient substrate

(ii)

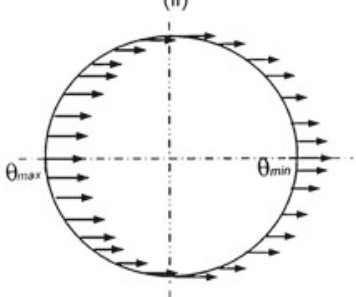

(ii)

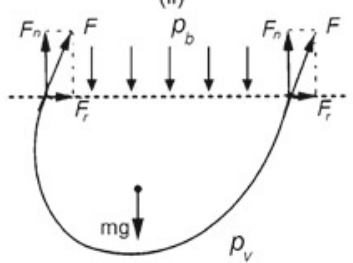

(iii)
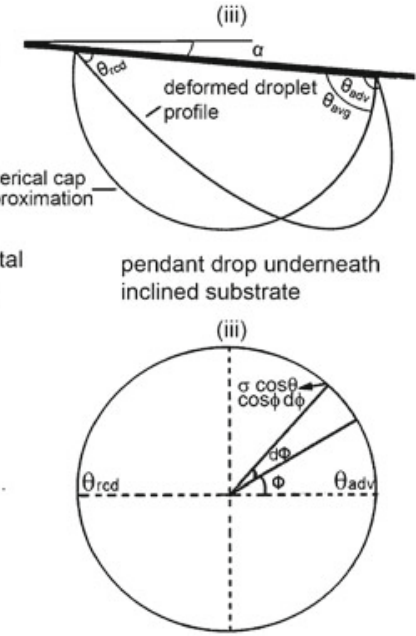

(iii)

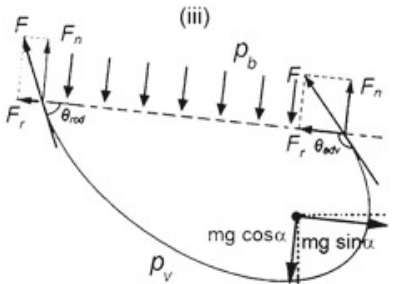

Figure 6. (a) Shape of a drop determined by a spherical cap approximation: (i) horizontal substrate without wettability gradient, (ii) horizontal substrate with wettability gradient, and (iii) inclined substrate. (b) Direction of retention force shown on the footprint of the drop; (c) free body diagram of a drop underneath planes with varying inclination.

a portion of a sphere of radius $r$ making a contact angle $\theta_{\text {avg }}(=\theta)$. From the geometry, the drop volume $V$, area of liquid-vapor interface $A_{l v}$, and area of solid-liquid interface $A_{s l}$, are given as

$$
\begin{gathered}
V=\left(\frac{\pi r^{3}}{3}\right) \cdot\left(2-3 \cos \theta_{\text {avg }}+\cos ^{3} \theta_{\text {avg }}\right) . \\
A_{l v}=2 \pi r^{2}\left(1-\cos \theta_{\text {avg }}\right) . \\
A_{s l}=2 \pi r^{2}\left(1-\cos ^{2} \theta_{\text {avg }}\right) .
\end{gathered}
$$

For a horizontal hydrophobic substrate, surface tension and gravity are in competition, figures $6 \mathrm{~b}-\mathrm{i}$ and $6 \mathrm{c}-\mathrm{i}$. As droplets grow in size, the body forces eventually surpass the limiting surface force on the three-phase contact line; the maximum base radius of a drop at instability is derived as

$$
\left(r_{b}\right)_{\max }=\sin ^{2} \theta_{\text {avg }} \sqrt{\frac{6 \sigma}{\Delta \rho g\left(2-3 \cos \theta_{\text {avg }}+\cos ^{3} \theta_{\text {avg }}\right)}} .
$$

Although the arguments leading to this equation do not include adhesion of the liquid with the substrate at the base, Leach et al (2006) have shown that such effects are of higher order and can be neglected for engineering calculations on the meso- and micro-scales. 
A horizontal substrate with wettability gradient is shown in figure 6a-ii. Here, drop motion can be expected even before the shapes are greatly altered by gravity or flow related pressure non-uniformity. Gravity and pressure will not have components parallel to the horizontal surface. Force imbalance is generated in this direction primarily because of variation of contact angle from $\theta_{\max }$ to $\theta_{\min }$ at the three-phase contact, arising from the substrate wettability gradient, figure $6 \mathrm{~b}-$ ii. For intermediate calculations, the drop shape is taken to be hemispherical in the sense that drop volume $V$, area of liquid-vapor interface $A_{l v}$, and area of solid-liquid interface $A_{s l}$ are calculated using the average contact angle $\theta_{\text {avg }}=\left(\theta_{\max }+\theta_{\min }\right) / 2$. If the weight of the drop is higher than the net retention force surface evaluated at the three-phase contact line in a direction normal to the surface, figure $6 \mathrm{c}-\mathrm{ii}$, the drop will fall-off at a critical radius, given by

$$
\left(r_{b}\right)_{\max }=\sqrt{\frac{3 \sigma \sin ^{3} \theta_{\text {avg }}\left(\sin \theta_{\max }+\sin \theta_{\min }\right)}{\left(\rho_{l}-\rho_{v}\right) g\left(2-3 \cos \theta_{\text {avg }}+\cos ^{3} \theta_{\text {avg }}\right)}} .
$$

Underneath an inclined hydrophobic substrate, figure 6a-iii, force imbalance is generated because of drop deformation arising from gravity and contact angle hysteresis. When the component of the gravitational force, with surface tension subtracted in the direction parallel to the substrate, balances wall shear, the drop will slide at a constant speed. The estimation of terminal velocity of a drop is discussed in a later section. The sliding of the drop wipes-off other drops that lie in its path, thus accumulating mass during motion. The contact angle hysteresis, namely the variation in the advancing to receding contact angle, is taken to vary linearly with respect to the azimuth angle along the three-phase contact line, as depicted in figure 6b-iii. The shape of three-phase contact line (foot print) of deformed drop is assumed to be circular. The force balance on a drop underneath an inclined substrate is shown in figure 6c-iii. As the drops grow, the gravity component of body force parallel to the substrate tries to slide the drop and surface tension provides the retention force to provide stability. Expressions for the maximum base radius of a drop that will first slide $\left(r_{\text {crit }}\right)$ or fall-off $\left(r_{\max }\right)$ underneath an inclined substrate are provided here. The critical radius of the droplet at slide-off on an inclined substrate is

$$
\left(r_{b}\right)_{c r i t}=\sqrt{\left(\frac{1.25 \sin ^{3} \theta_{a v g}}{\left(2-3 \cos \theta_{a v g}+\cos ^{3} \theta_{a v g}\right)}\right)\left(\cos \theta_{r c d}-\cos \theta_{a d v}\right)\left(\frac{\sigma}{(g \sin \alpha)\left(\rho_{l}-\rho_{v}\right)}\right)} .
$$

The maximum radius of the drop that will initiate fall-off is obtained by examining forces perpendicular to substrate as

$$
\left(r_{b}\right)_{\max }=\sqrt{\left(\frac{3\left(\sin ^{3} \theta_{a v g}\right)\left(\sin \theta_{r c d}+\sin \theta_{a d v}\right)}{\left(2-3 \cos \theta_{a v g}+\cos ^{3} \theta_{a v g}\right)}\right)\left(\frac{\sigma}{(g \cos \alpha)\left(\rho_{l}-\rho_{v}\right)}\right)} .
$$

2.2e Modelling of flow and heat transfer within a sliding drop: It is assumed here that the sliding drop attains terminal velocity instantaneously after the incipience of instability. The determination of terminal velocity is discussed in the following section. Although drop movement further enhances heat transfer coefficient, the long term sustainability of the condensation process is adversely affected because of the possibility of leaching the promoter layer of the substrate. Therefore, from a modelling perspective, the terminal velocity and the wall shear stress 
are quantities of importance that relate to the deterioration/leaching of the promoter layer. In the present section, the calculation of the skin friction coefficient and Nusselt number is discussed.

The schematic diagram of a three-dimensional deformed liquid drop, with an advancing angle $\theta_{a d v}$ and a receding angle $\theta_{r c d}$, is shown in figure 7a. The drop is deformed and the difference in angles between the advancing (leading) and receding (trailing) sides is the contact angle hysteresis. Simulations have been carried out with contact angle as a parameter. For horizontal surfaces, the shape of the drop is taken to be a part of a sphere. On inclined surfaces, the advancing and receding angles are utilized to obtain the shape of the drop by the two-circle approximation (Elsherbine \& Jacobi 2006; Sikarwar et al 2011c).

The frame of reference for flow and transport calculations is fixed within the liquid drop, the wall moving relative to it at constant speed. A single drop, discretized using tetrahedral elements, is shown in figure 7b. Fluid flow and temperature distribution are obtained by numerically solving the unsteady Navier-Stokes equations and energy equations in three-dimensions. The numerical simulation is based on collocated finite volume discretization of the unsteady three-dimensional Navier-Stokes equations over an unstructured mesh of tetrahedral elements of nearly equal volumes (Date 2005). Pressure-velocity coupling is treated using a smoothing pressure correction method that results in a SIMPLE-like algorithm. Convective terms are discretized by a hybrid upwind scheme. The diffusion terms are discretized using a $2^{\text {nd }}$ order

(a)

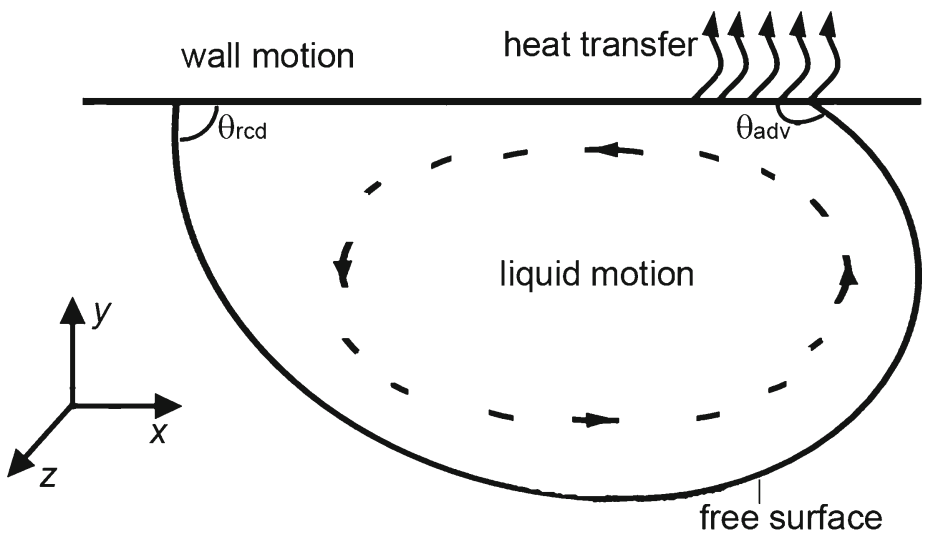

(b)

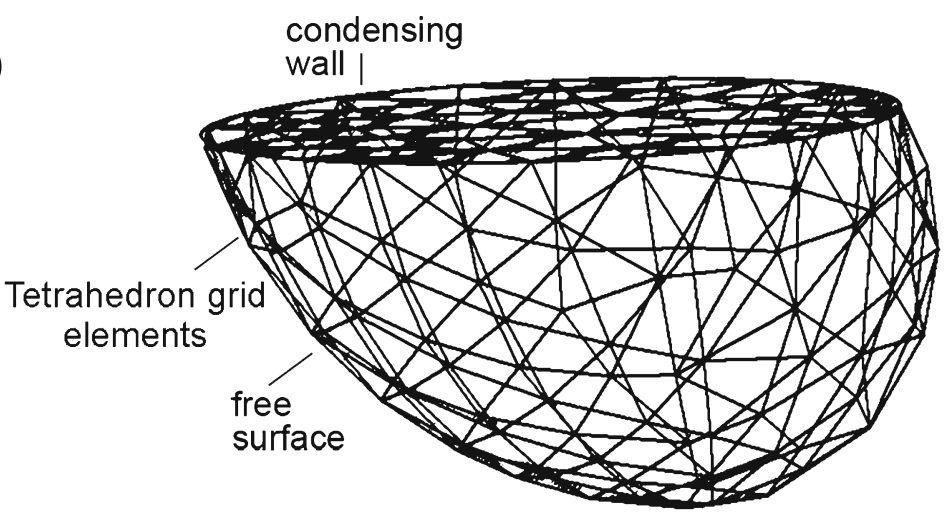

Figure 7. (a) Schematic diagram of fluid motion and heat transfer at the scale of a single drop; (b) Computational domain with tetrahedral elements in an unstructured grid. 
central-difference scheme. Geometry invariant features of the tetrahedral element are used so that the calculation of gradients at cell faces is simplified using nodal quantities of a particular variable. Nodal quantities, in turn, are calculated as a weighted average of the surrounding cellcentered values (Barth \& Jespersen 1989; Frink et al 1991). The discretized system of algebraic equations is solved by the stabilized bi-conjugate gradient method (biCGStab) with a diagonal pre-conditioner. The overall numerical algorithm is similar to that proposed by Date (2005). Points of difference are related to the use of certain invariant properties of the tetrahedral element, powerful linear equations solver as well as a parallel implementation of the computer program. Iterations within the code are run till an absolute convergence of $10^{-7}$ is reached in the residuals. Calculations progress till steady-state is reached (Sikarwar et al 2013).

Figure 8a shows streamlines and dimensionless velocity contours within a drop for various Reynolds numbers. Two cross-sectional planes of the drop have been considered. Dimensionless temperature contours are also shown with water as the condensing fluid, figure $8 \mathrm{~b}$. From the

(a)

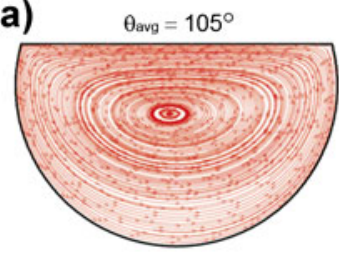

(b)

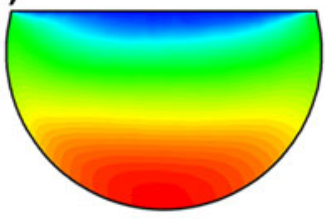

(c)

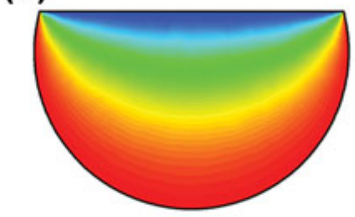

(d)

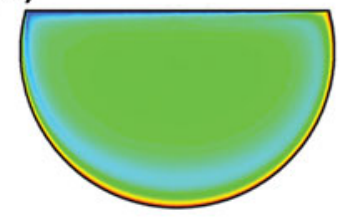

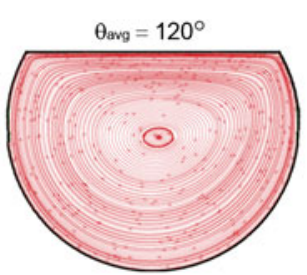

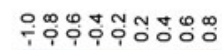

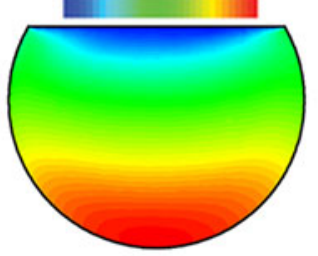

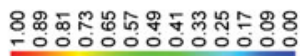
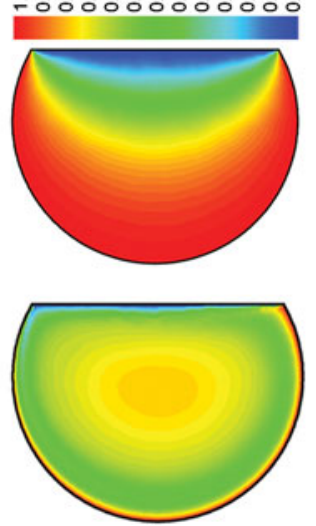
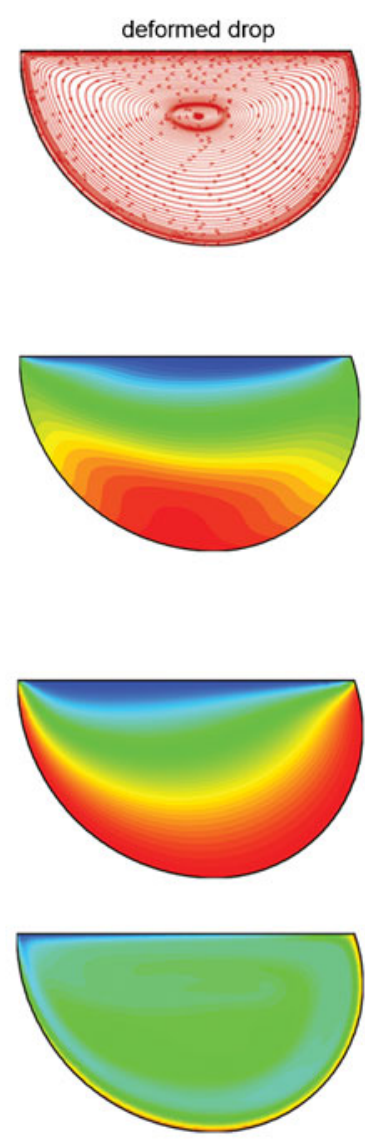

Figure 8. (a) Streamlines showing the motion of the liquid inside the sliding drop of water at a Reynolds number of 1000; (b) contours of dimensionless $x$-component velocity at $\mathrm{Re}=1000$ on the plane considered in (a); (c) dimensionless temperature contours in water under no-flow conditions; (d) dimensionless temperature contours in water at $\operatorname{Re}=1000$. Symmetric drops with contact angles of 105 and $120^{\circ}$ are considered. The deformed drop has an advancing angle of $109^{\circ}$ and a receding angle of $98^{\circ}$. 
velocity and temperature data, wall shear stress and heat transfer rates are determined. The wall shear stress and heat flux distribution at the base of the drop are shown in figures 9a and $\mathrm{b}$. Correlations in terms of skin friction coefficient and Nusselt number that include contact angle as a parameter are presented in figure 10 for various Prandtl numbers. The resulting correlations, that serve as input to the overall condensation model, are summarized as

$$
\overline{\mathrm{C}_{f}}=58 \mathrm{Re}^{-0.97} \theta_{\text {avg }}^{-1.58} .
$$
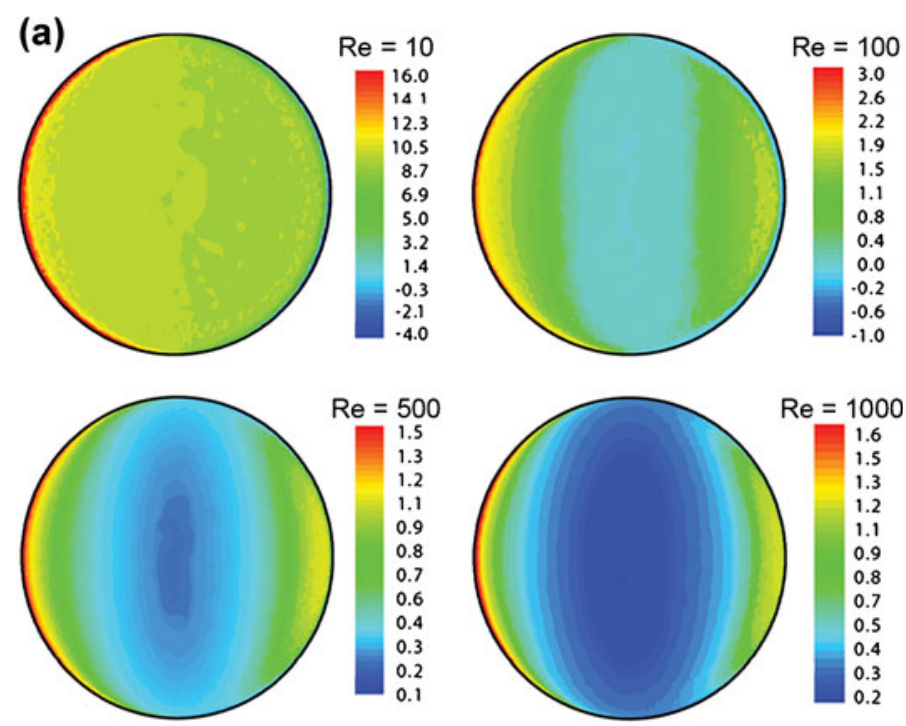

(b)
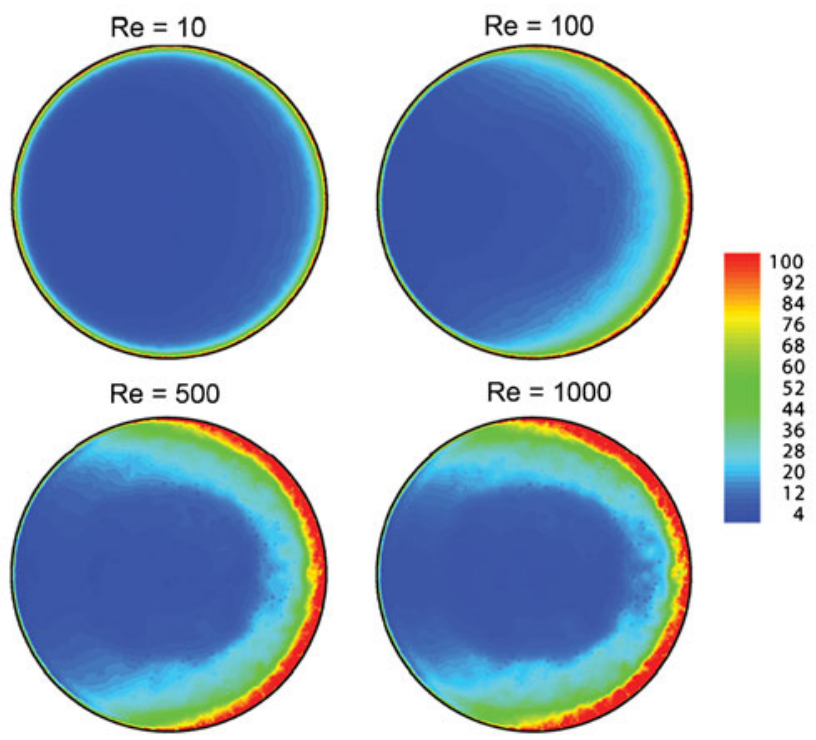

Figure 9. (a) Wall shear stress and (b) nusselt number distribution for a deformed water drop at various Reynolds numbers. The deformed drop has an advancing angle of $109^{\circ}$ and a receding angle of $98^{\circ}$. 
(a)

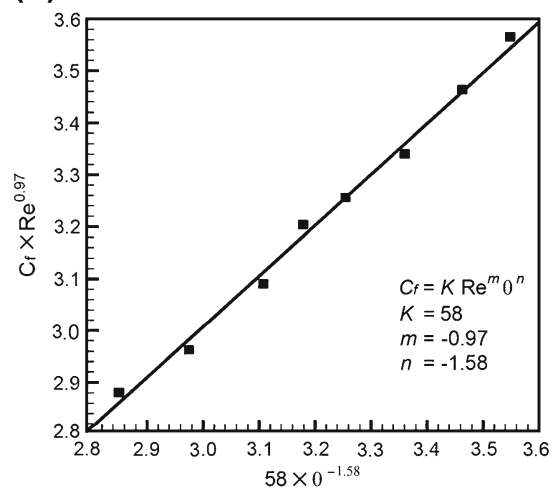

(b)

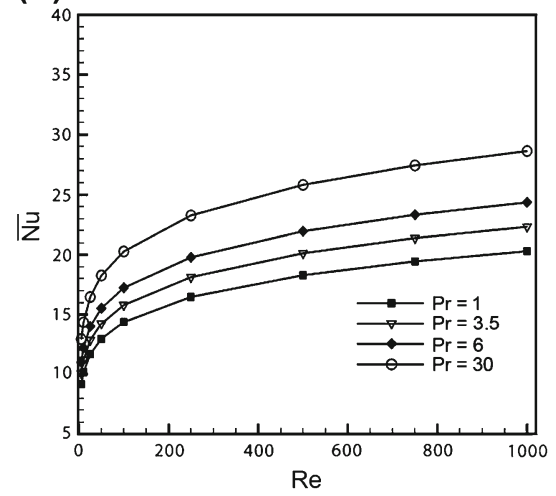

Figure 10. (a) Numerical correlation of a composite product of skin friction coefficient and Reynolds number as a function of the average contact angle. (b) Variation of average Nusselt number with Reynolds number of a sliding drop for various Prandtl number fluids. Symmetric as well as deformed drops are considered.

$$
\begin{gathered}
\text { For } \operatorname{Pr}>1 \quad \overline{\mathrm{Nu}}=8.12 \operatorname{Re}^{0.20} \operatorname{Pr}^{0.1} \theta_{\text {avg }}^{-0.8} \\
\text { For } \operatorname{Pr}<1 \quad \overline{\mathrm{Nu}}=18.47 \theta_{\text {avg }}^{-1.26} .
\end{gathered}
$$

Here, $\theta_{\text {avg }}$ is in radians. For low Prandtl number fluids such as liquid metals, convection effects are overwhelmed by diffusion and Nusselt number becomes practically independent of Reynolds number.

2.2f Calculation of terminal velocity: As has been discussed in dropwise condensation, droplets undergo instability and start sliding over the substrate that is either inclined or is horizontal but with a wettability gradient. The speed increases with time till the unbalanced force is matched by wall shear, subsequently resulting in a constant terminal velocity. In the present model, it is assumed that drops attain terminal velocity immediately after the instability condition. The calculation of terminal velocity is discussed below.

\section{(a) Horizontal substrate with a wettability gradient}

The wettability gradient, which facilitates passive drop motion, is shown in figure 11a. The contact angle at the non-wetting side $(x=0)$ is taken as $\theta_{1}$, while that at the higher wettability side $(x=X)$ is $\theta_{2}$. The local apparent contact angle which the contact line will make is taken to vary linearly in one direction from $x=0$ to $X$, as follows

$$
\theta=\theta_{1}-\left(\frac{\theta_{1}-\theta_{2}}{X}\right) \cdot x
$$

In a time-marching calculation, drops grow in size and are subjected to increasing values of gravitational force as well as the driving force due to contact angle hysteresis. For a horizontal 

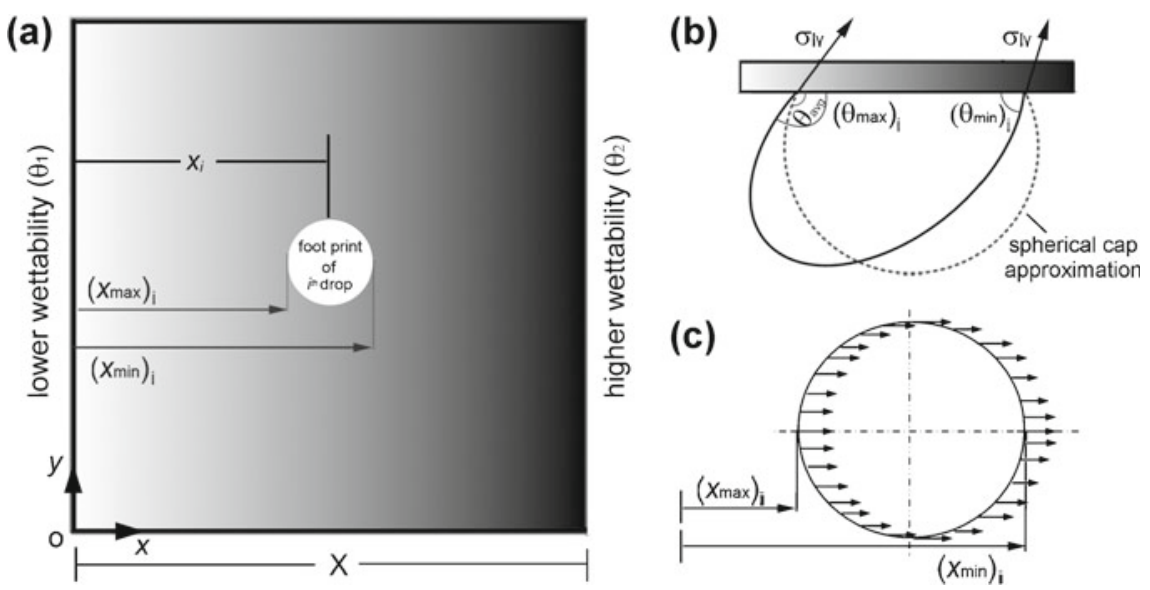

Figure 11. (a) Condensation over a substrate with wettability gradient. The footprint of the $i^{\text {th }}$ drop is assumed to be circular. (b) Side view of each drop is determined by the two circle approximation. (c) Direction of forces acting over the three phase contact line, namely the substrate-vapor-liquid boundary.

substrate, sliding is initiated by a resultant surface tension along the three-phase contact line. The base radius of the drop and the contact angle difference between the trailing and leading edge is calculated at each time step, figure $11 \mathrm{~b}$. The retention force exerted by the substrate on the drop at the contact line due to wettability gradient is calculated. In this calculation, the footprint of the drop is assumed to be a circle, figure 11c. The calculation steps within the time marching algorithm are as follows

(i) The base radius of droplet of $i^{\text {th }}$ is determined as

$$
\left(r_{b}\right)_{i}=\left[r_{i}^{\text {old }}+\left(r_{i}^{\text {new }}-r_{i}^{\text {old }}\right)\right] \sin \left(\theta_{\text {avg }}\right)_{i}
$$

(ii) Calculate the $x_{\min }$ and $x_{\max }$ for the $i^{\text {th }}$ drop. After knowing these values, $\left(\theta_{\max }\right)_{i}$ and $\left(\theta_{\min }\right)_{i}$ are calculated as

$$
\begin{aligned}
& \left(\theta_{\max }\right)_{i}=\theta_{1}-\left(\frac{\theta_{1}-\theta_{2}}{X}\right)\left(x_{\max }\right)_{i} . \\
& \left(\theta_{\min }\right)_{i}=\theta_{1}-\left(\frac{\theta_{1}-\theta_{2}}{X}\right)\left(x_{\min }\right)_{i} .
\end{aligned}
$$

(iii) The variation of contact angle at footprint of the $i^{\text {th }}$ droplet is given by

$$
\left(\theta_{x}\right)_{i}=\left(\theta_{\max }\right)_{i}+\left(\frac{\left(\theta_{\min }\right)_{i}+\left(\theta_{\max }\right)_{i}}{\left(x_{\min }\right)_{i}-\left(x_{\max }\right)_{i}}\right) x_{i} .
$$

Here, $x_{i}$ is the center coordinates of the $i^{\text {th }}$ drop, as shown in figure 11 . 
(iv) The net force acting in the direction of velocity, for the drop being analysed, is given by,

$$
\left(F_{r}\right)_{i}=2 \pi \sigma_{l v}\left(r_{b}\right)_{i} \int_{\left(x_{\max }\right)_{i}}^{\left(x_{\min }\right)_{i}} \cos \left(\theta_{x}\right)_{i} d x_{i}
$$

Hence, the force $\left(F_{r}\right)$ responsible for mobilizing the $i^{\text {th }}$ drop is

$$
\left(F_{r}\right)_{i}=(4 / \pi) \sigma\left(r_{b}\right)_{i}\left[\cos \left(\theta_{\min }\right)_{i}-\cos \left(\theta_{\max }\right)_{i}\right] .
$$

The wall shear that resists drop motion is estimated as

$$
\left(F_{h y d}\right)_{i}=\mathrm{C}_{f}\left(0.5 \rho U_{i}^{2}\right)\left(A_{s l}\right)_{i} .
$$

The coefficient of skin friction $\mathrm{C}_{f}$ is obtained from the correlation (Eq. 20) derived from the CFD model described earlier. Equating the expressions of forces given by Equations (28) and (29), the terminal velocity of the $i^{\text {th }}$ drop is obtained as

$$
U_{i}=\left[\frac{0.044 \cdot\left(F_{r}\right)_{i}\left(\theta_{\text {avg }}\right)_{i}^{1.58}}{\rho^{0.03} \mu^{0.97}\left(d_{b}^{1.03}\right)_{i}}\right]^{1 / 1.03}
$$

Velocity, thus obtained, is a function of the drop size and position of the drop over the substrate having a wettability gradient.

\section{(b) Inclined substrate}

For an inclined substrate, the drop achieves terminal velocity when the component of weight parallel to the surface, retention force of the deformed drop at the three-phase contact line owing to surface tension, and wall friction are in balance, viz.,

$$
F_{g_{\|}}+F_{r}+F_{s}=0
$$

where, $F_{g_{\|}}$is the component of weight parallel to the inclined substrate, $F_{r}$ is the retention force opposing drop motion and $F_{S}$ is wall shear associated with the relative velocity between the fluid and the substrate. These force components are respectively written as

$$
\begin{gathered}
F_{g \|}=\frac{\pi}{3 \sin ^{3} \theta_{a v g}} r_{b}^{3}\left(2-3 \cos \theta_{a v g}+\cos ^{3} \theta_{a v g}\right) \rho_{l} g \sin \alpha \\
F_{r}=2 \int_{0}^{\pi} \sigma \cos \theta \cos \phi r_{b} d \phi .
\end{gathered}
$$

Here, the contact angle $\theta$ is a linear function of the azimuthal angle $\phi$. Therefore, the retention and wall shear forces are given as

$$
\begin{gathered}
F_{r}=(4 / \pi) r_{b} \sigma\left(\cos \theta_{r c d}-\cos \theta_{a d v}\right) \\
F_{s}=(1 / 2) C_{f} A_{s l} \rho U^{2} .
\end{gathered}
$$


Once again, the average skin friction coefficient $\left(C_{f}\right)$ is determined at the scale of the individual drop by a CFD model as described earlier. The terminal velocity of the drop over an inclined surface that makes an angle $\alpha$ with the horizontal can now be calculated as

$$
U=\sqrt{\frac{2\left(F_{g \|}-F_{r}\right)}{C_{f} \rho A_{s l}}} .
$$

(a)

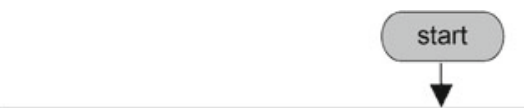

enter the fluid properties, substrate size, number of nucleation sites, substrate orientation, total time of condensation, time step and type of substrate

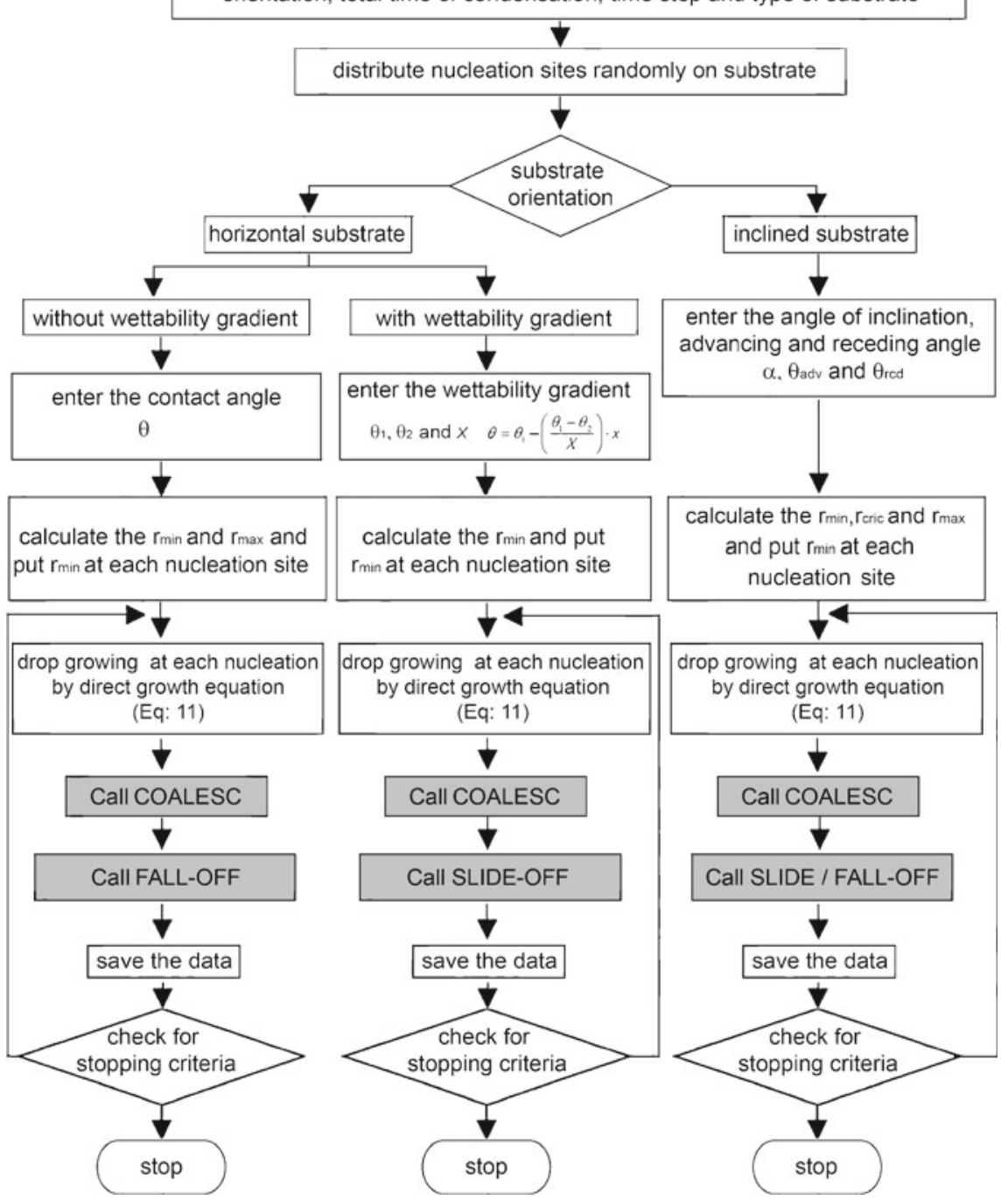

Figure 12. (a) Flow diagram of the macroscale dropwise condensation model. (b) Flow diagram of subroutines used in the macroscale dropwise condensation model. 
2.2g Wall heat transfer rates: Heat transfer during dropwise condensation can be calculated from rate of condensation at the free surface of the drop at each nucleation site of the substrate. The gaps between drops are assumed to be inactive for heat transfer. The heat transfer rate $q$ is a function of the nucleation site density and the rate of growth of drop radius at each nucleation site. The latter is estimated by using a quasi one-dimensional approximation for thermal resistances, including the interfacial and capillary resistance at the vapor-liquid boundary and conduction resistance through drop, as discussed in section $2.2 \mathrm{~b}$ and given by equation (11). The rate of condensation at each nucleation site can now be determined as follows.

The mass of condensate accumulated at the $i^{\text {th }}$ nucleation site over a time interval $\Delta t$ is calculated as

$$
(\Delta m)_{i}=\rho \frac{\pi}{3}\left(2-3 \cos \theta_{\text {avg }}+\cos ^{3} \theta_{\text {avg }}\right)\left(r_{\text {new }}^{3}-r_{\text {old }}^{3}\right)_{i} .
$$

(b)
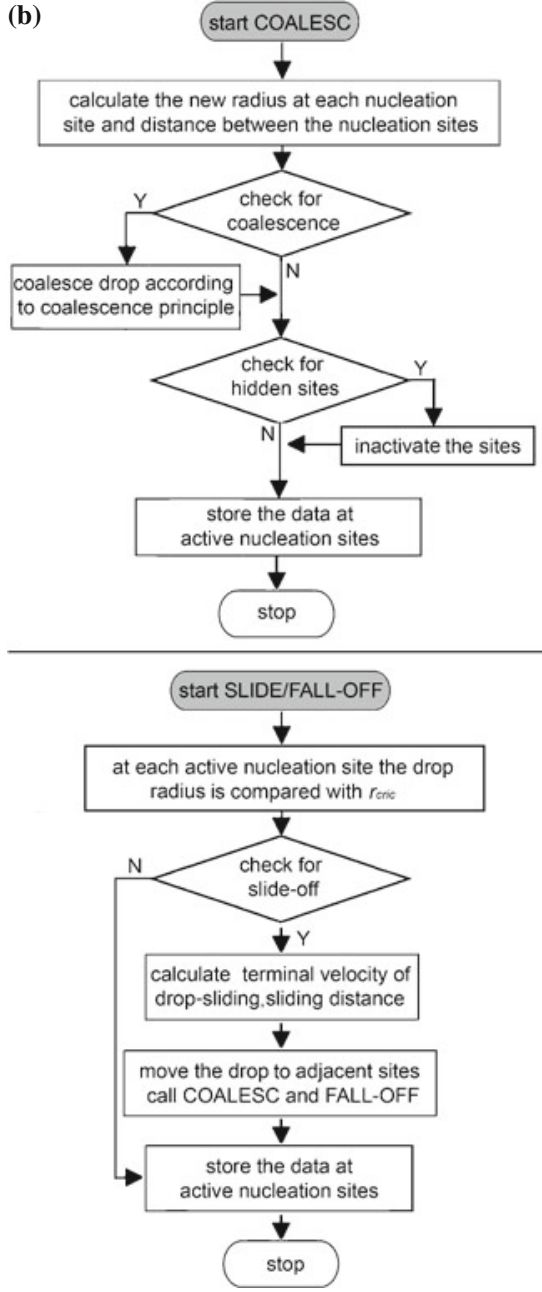

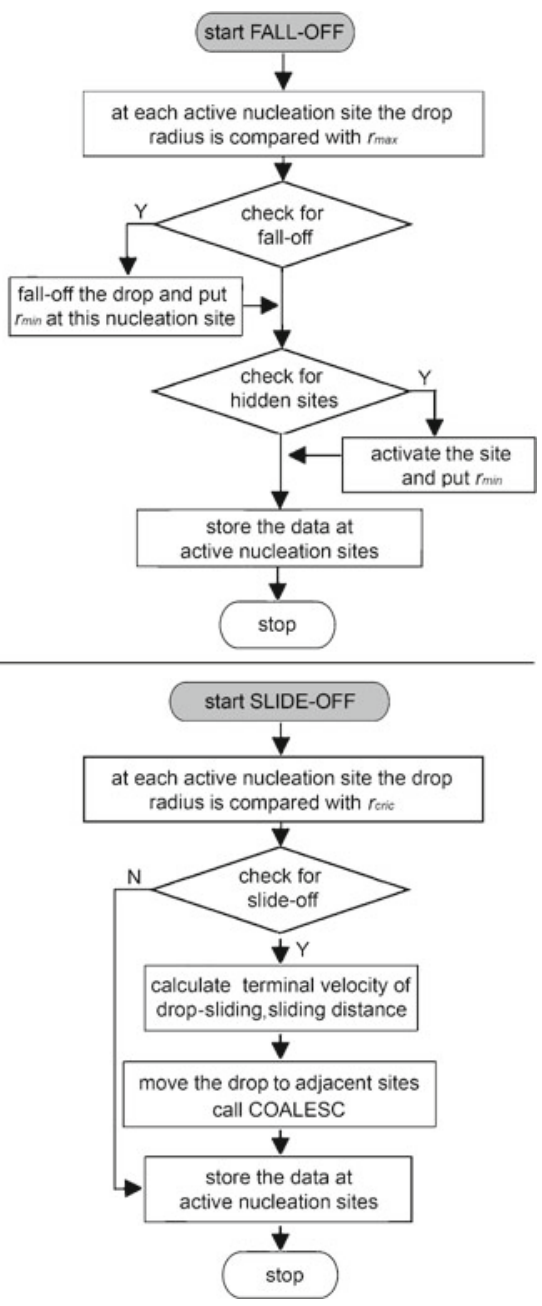

Figure 12. (continued). 


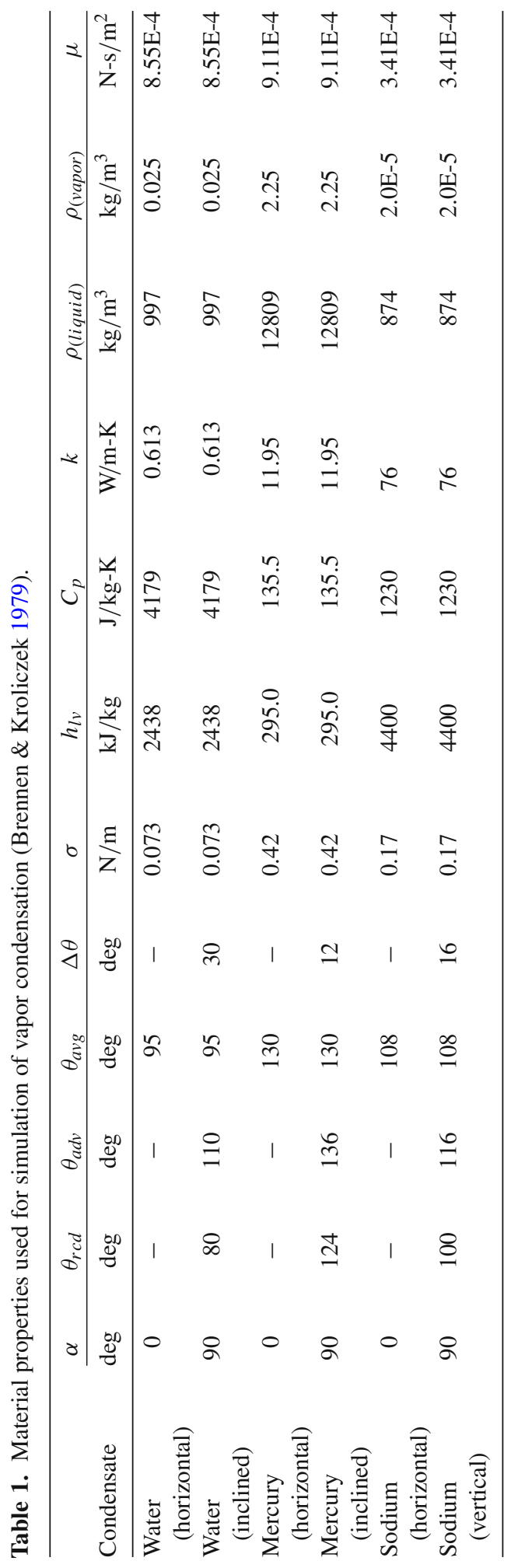


With $N$, the number of active nucleation sites, the total quantity of condensate at given time step $(\Delta t)$ is obtained as

$$
\Delta m=\sum_{i=1}^{i=N}(\Delta m)_{i}
$$

Therefore, the average rate of condensation underneath a substrate is:

$$
(m)_{a v g}=\frac{\sum_{j=1}^{j=K}(\Delta m)_{j}}{t} \text { where } t=\left(\sum_{j}^{j=K}(\Delta t)_{j}\right) \text {. }
$$

Here, $t$ is the time period of condensation and $K=(t / \Delta t)$ is the number of time steps. The heat transfer rate is simply the latent heat release during condensation divided by the time elapsed. The sensible cooling of the liquid is neglected. The average heat transfer coefficient over an area $(A)$ of the substrate during dropwise condensation is then given by

$$
\overline{h_{c}}=\frac{m_{a v g} h_{l v}}{A\left(T_{s a t}-T_{w}\right)} .
$$
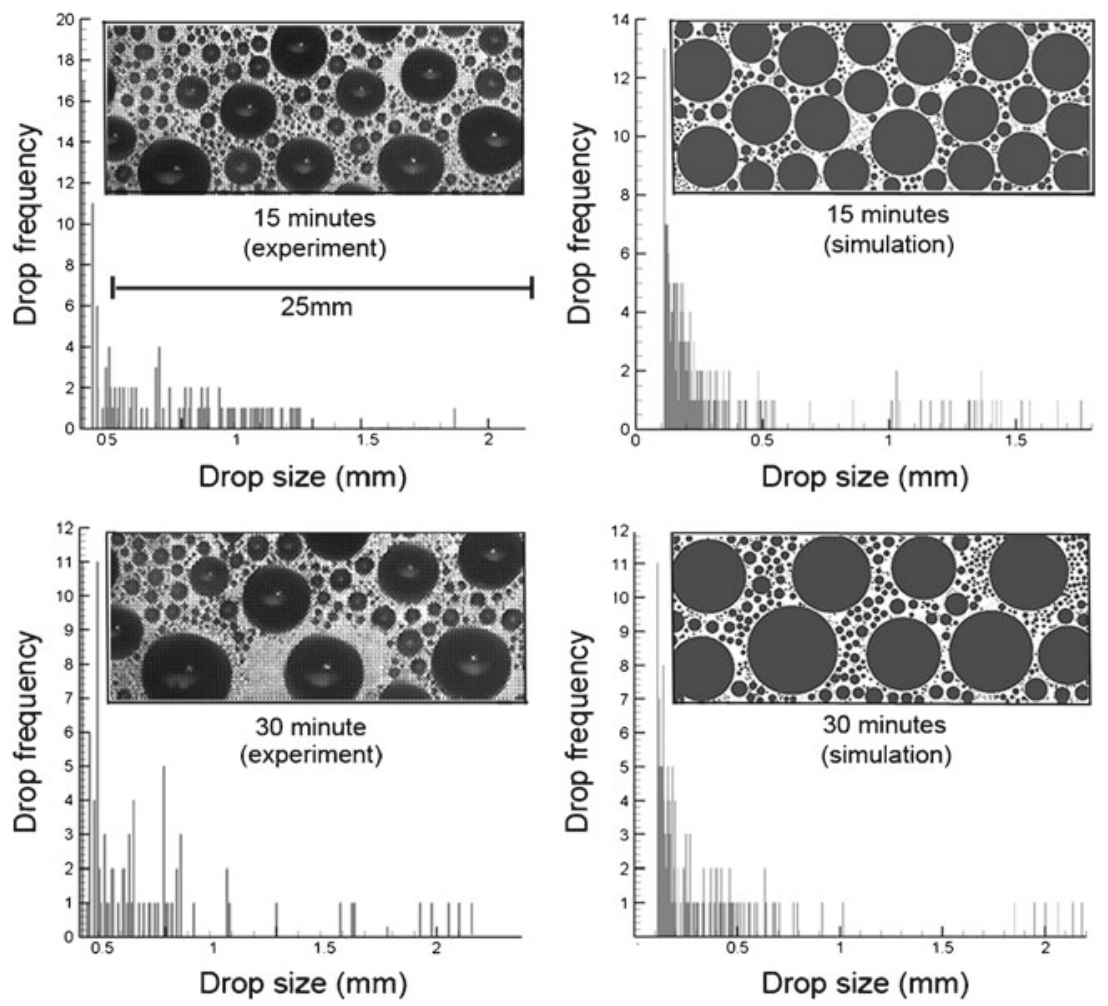

Figure 13. Visual comparison of spatial drop distribution patterns in experiments and simulation and the corresponding histograms of drop sizes when dynamic steady state is reached (Sikarwar et al 2011a). A horizontal surface is considered where water condenses on the underside; $T_{\text {sat }}=27^{\circ} \mathrm{C}, \Delta T=5^{\circ} \mathrm{C}$ and apparent contact angle $=95^{\circ}$. 


\subsection{Numerical algorithm of the complete dropwise condensation model}

The important steps in the numerical algorithm are now stated as follows.

(i) Initialize all variables and provide material properties.

(ii) Distribute the nucleation sites on the substrate using a random number generator and place the drop of minimum radius at all nucleation sites.
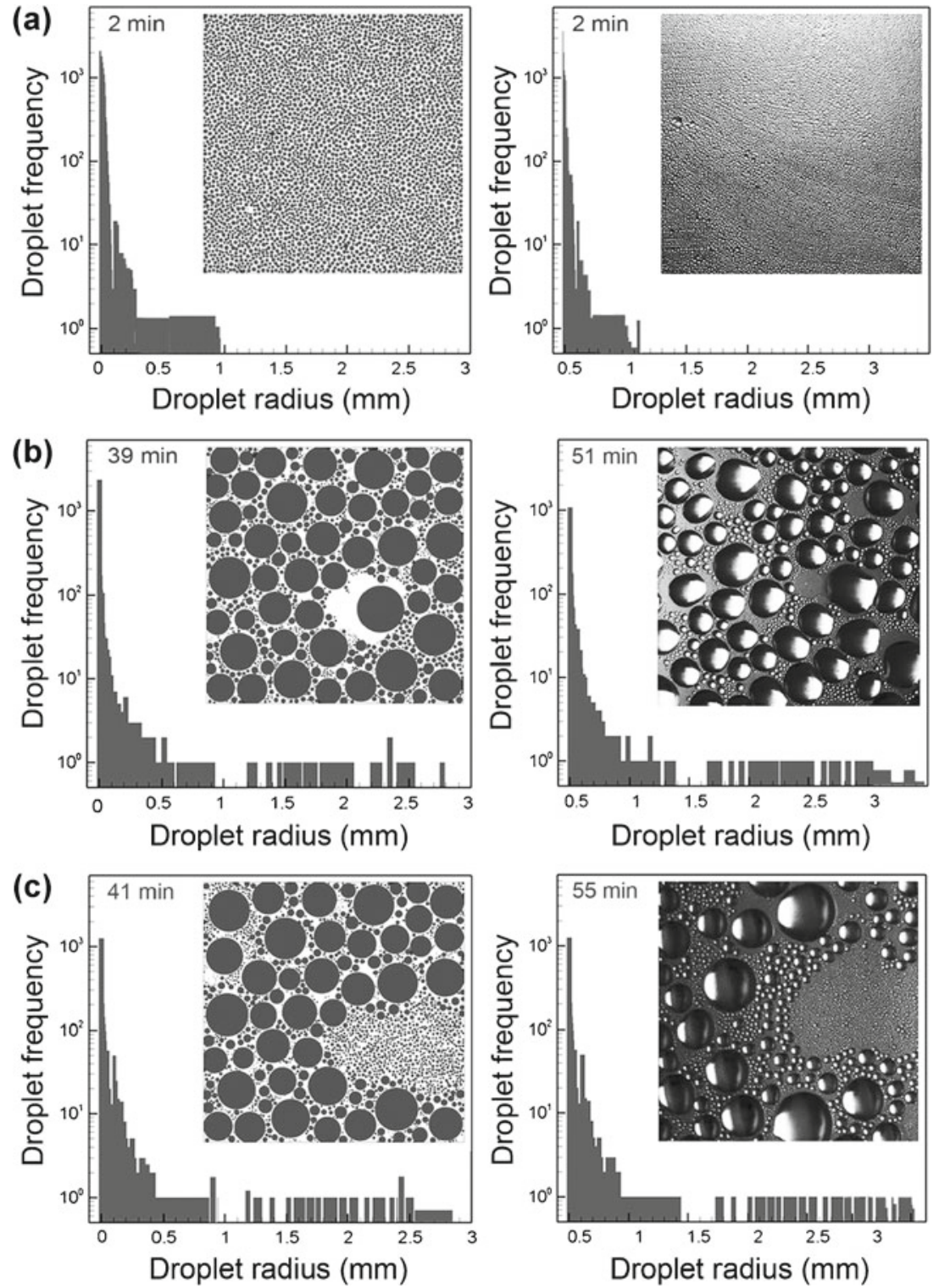

Figure 14. Size distribution of water drops condensing underneath a silanized glass substrate inclined at an angle of $15^{\circ}$, as recorded in experiments and in simulations (Sikarwar et al 2011a); $T_{\text {sat }}=27^{\circ} \mathrm{C}$, $\Delta T=5^{\circ} \mathrm{C}$ and contact angle hysteresis $=30^{\circ}$. 
(iii) Solve Equation (11) by a $4^{\text {th }}$ order Runge-Kutta method and find the new radius.

(iv) Calculate the intermediate distance between nucleation sites.

(v) Check for coalescence.

(vi) Identify the nucleation sites covered by the resulting coalesced drops and keep them deactivated till the drop is covering them.

(vii) Simultaneously, search for newly exposed sites created due to drop coalescence and provide a minimum radius drop on such newly exposed sites.

(viii) For all the drops, check for the critical radius of slide-off and the sliding velocity.

(ix) Re-activate the exposed sites created due to drop slide-off and provide a minimum radius drop on such newly exposed sites.

(x) Check for drop fall-off.

(xi) Re-activate the exposed sites created due to drop fall-off and provide a minimum radius drop on such newly exposed sites.

(xii) Repeat (iii)-(ix) till a dynamic steady-state is reached.

The flow chart of the mathematical model of dropwise condensation underneath inclined substrates is depicted in figure 12a, while the subroutines are detailed is in figure $12 \mathrm{~b}$. Whenever a drop is removed or shifted from its location due to slide and coalescence, all hidden nucleation sites underneath the drop become active and are immediately supplied with thermodynamically stable droplets of minimum radius. It is to be noted that the simulation needs to track multiple generations of the droplets - nucleating, growing by direct condensation, by coalescence and some slide/falling-off, when the virgin surface thus exposed, is re-nucleated. The computations are hence, quite intensive.

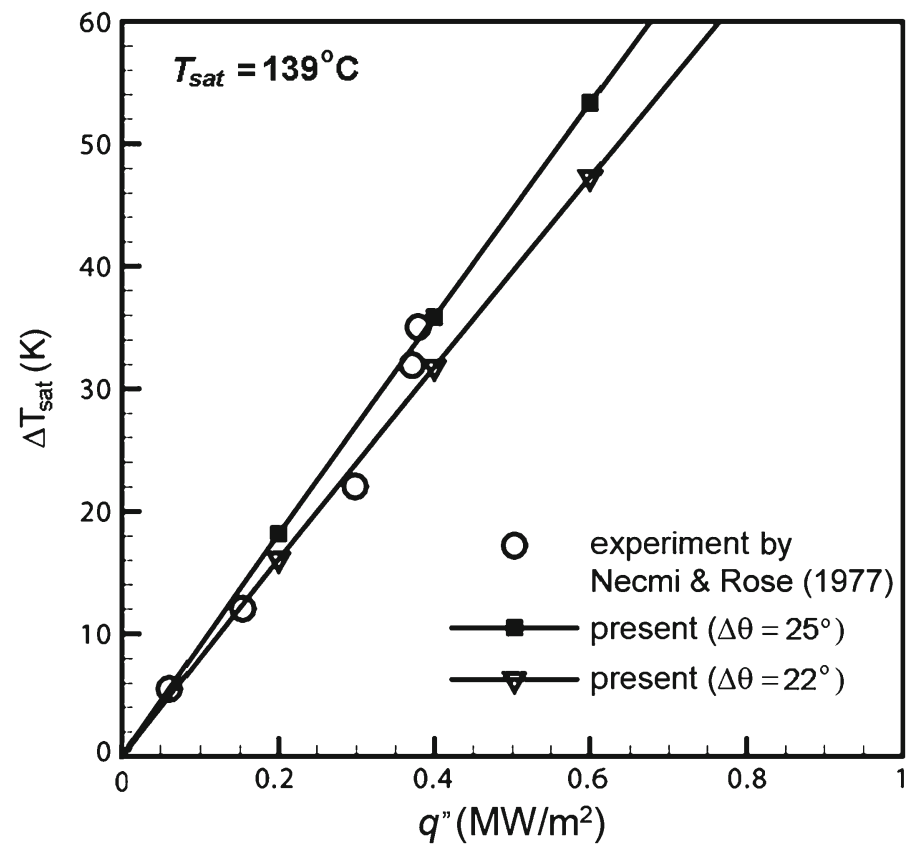

Figure 15. Average substrate heat flux $\left(q^{\prime \prime}\right)$ plotted as a function of degree of sub-cooling $\left(\Delta T_{\text {sat }}\right)$ for dropwise condensation of mercury over a vertical plate at saturation temperature of $139^{\circ} \mathrm{C}$ and contact angle hysteresis $(\Delta \theta)=22^{\circ}$ and $25^{\circ}$. 
While the mathematical model developed is quite general, simulations have been carried out under the following assumptions.

1. Nucleation sites are randomly distributed on the surface. Unless stated otherwise, all computations have been performed with an initial nucleation site density of $10^{6} \mathrm{~cm}^{-2}$.

2. Thermodynamically constrained smallest radius is taken as the minimum radius in the simulation. Initially, the substrate is virgin and all nucleation sites are instantaneously occupied by the droplet of minimum radius.

3. Heat transfer resistance arises due to the liquid-vapor interface, curvature and conduction, driven by imposed sub-cooling of the substrate. Convective transport of heat is neglected for static drops but is included for a drop in motion. Constriction resistance is neglected.

4. The accommodation coefficient is taken to be 0.02 for water and 0.21 for sodium (Carey 1992). Literature suggests that accommodation coefficient is also a function of temperature, but is not considered in the present simulation.

5. Droplet coalescence is assumed to be instantaneous and resulting droplet attains instantaneous mechanical stability; interface oscillations are neglected. Also, any inertial effect and change of shape of the drop due to acceleration are neglected.

(a)

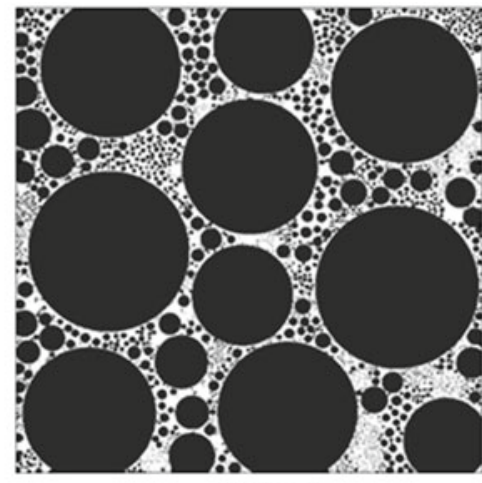

sodium

(b)

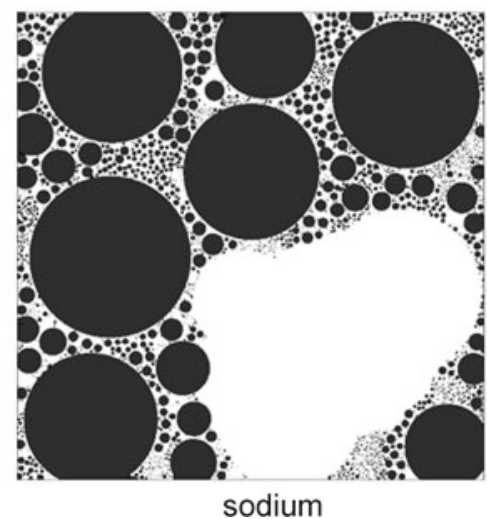

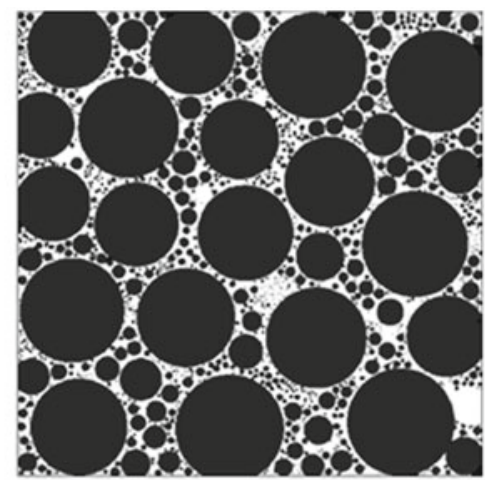

water

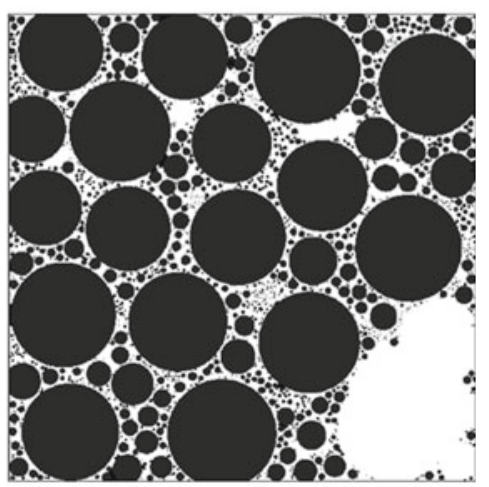

water

Figure 16. Spatial drop distribution of liquid sodium compared with water in dropwise condensation underneath a horizontal substrate; time instants considered are (a) just before fall-off and (b) just after fall-off. 
6. An equivalent spherical-cap approximation has been incorporated to model droplet shapes. For drops on inclined surfaces, the two-circle approximation is used (Elsherbine \& Jacobi 2006).

7. Though contact angles are obtained (from theory or experiments) under static conditions, the same values have been used under dynamic conditions too.

8. Partial fall-off of drops is neglected in the sense that instability results in the complete volume of the drop being removed.

9. The entire substrate is assumed to be at constant temperature; drop motion leads to changes in the wall heat flux; local wall temperature fluctuations which may be present, as observed by Bansal et al (2009), have been neglected.

10. Thermophysical properties of the vapor and liquid phases are taken to be independent of temperature; the vapor is saturated; all properties are evaluated at the average of the substrate and saturated vapor temperatures.

Extensive parametric studies and independent validations of the above model with published data as well as experimental results have been reported in the recent past (Sikarwar et al 2011a, b, 2012). Important results derived from model simulation are discussed in the next section. Thermophysical properties of water and liquid metals are summarized in table 1 (Brennen \& Kroliczek 1979).

\subsection{Dropwise condensation patterns}

Experimental results of condensation patterns and the corresponding predictions of numerical simulation for water vapor at a saturation temperature of $27^{\circ} \mathrm{C}$ and subcooling of $5^{\circ} \mathrm{C}$ are first compared. Figure 13 depicts drops of various sizes formed during condensation underneath a

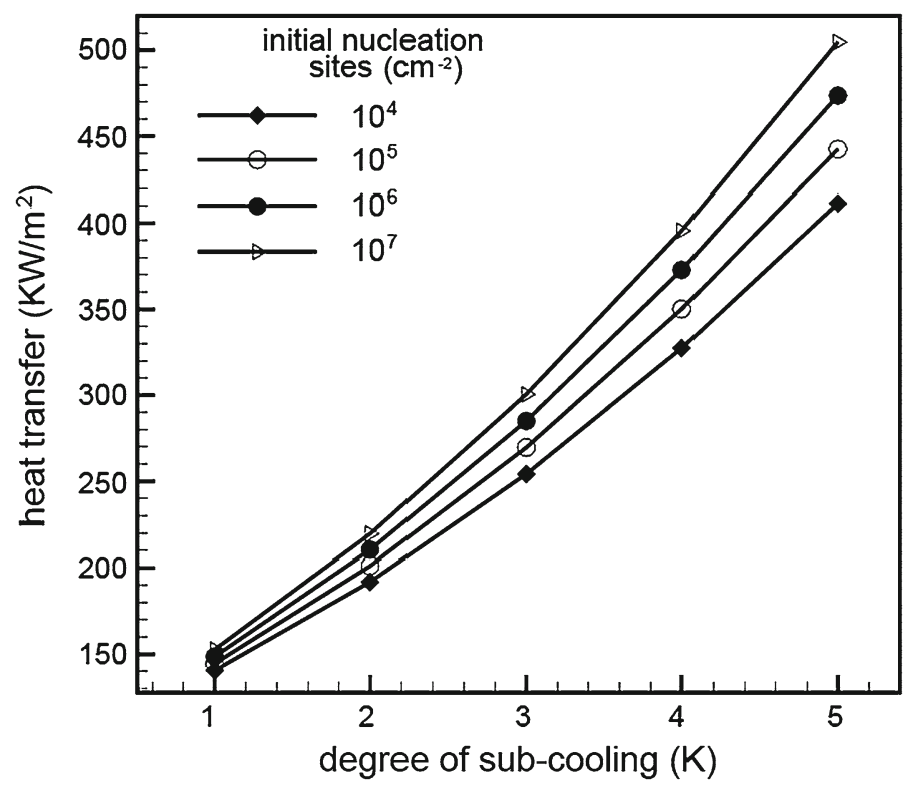

Figure 17. Overall heat transfer as a function of the degree of sub-cooling with nucleation site density as a parameter. Fluid considered is water at $300 \mathrm{~K}$ and a contact angle of $95^{\circ}$. 
horizontal substrate at 15 and 30 minutes, after the commencement of the condensation process. Figure 14 shows condensation patterns formed underneath a hydrophobic substrate inclined at an angle of $15^{\circ}$ to the horizontal (advancing angle $110^{\circ}$ and receding angle $80^{\circ}$ ). Drop distribution over the surface along with the histogram of drop sizes at a selected time instant is shown, both from experiments and simulation. The prediction of the model is in fair agreement with the experiment data of condensation of water vapor. The model results are also validated against experiments for condensation of mercury vapor on a vertical surface (figure 15). The experimental data for the surface-averaged wall heat flux as a function of the vapor to surface temperature difference is adopted from the work of Necmi \& Rose (1977). Figure 15 shows good overall agreement for all levels of subcooling. Hence, one may conclude that the model is robust and applicable to liquids, having a wide range of Prandtl numbers, condensing on substrates with different surface orientations and conditions.

2.4a Parametric studies: A parametric study as a function of choice of the condensing fluid, plate inclination, contact angle and contact angle hysteresis is now reported. Figure 16 shows drop distribution underneath a horizontal substrate, just before fall-off, of the largest drop and

(a)

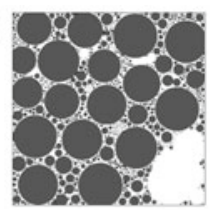

(i) horizontal: no gradient

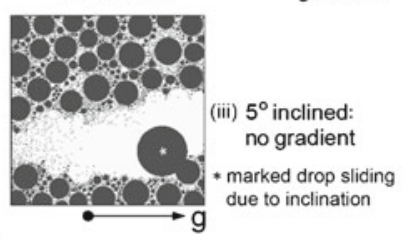

(c)

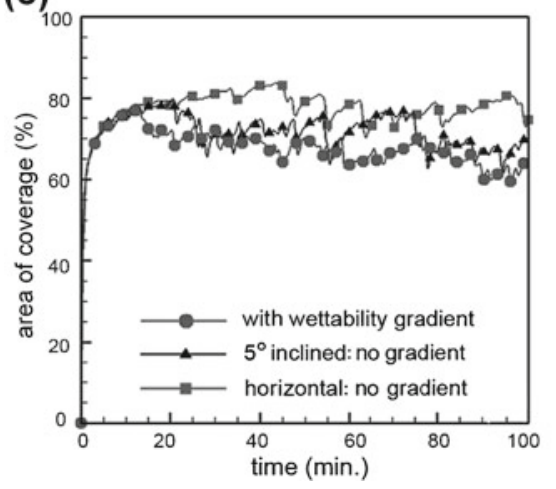

(b)

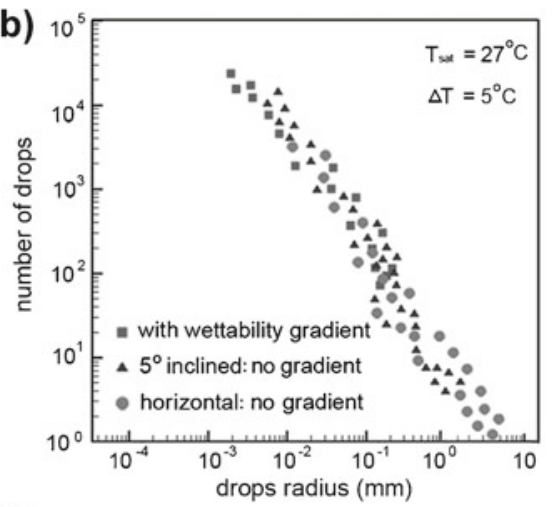

(d)

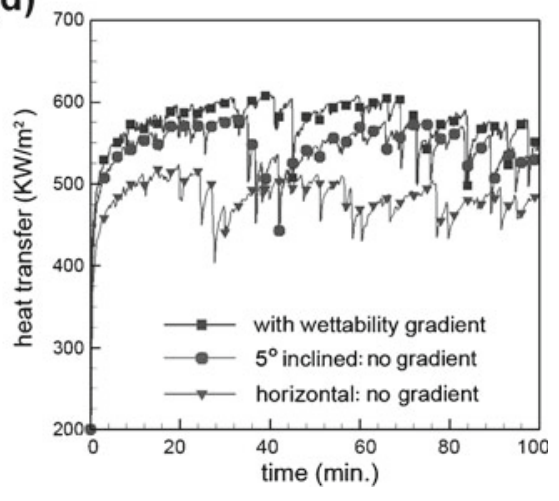

Figure 18. Dropwise condensation of water vapor at $27^{\circ} \mathrm{C}$ with sub-cooling of $\Delta T=5^{\circ} \mathrm{C}$ underneath surfaces of various textures. (a) Drop distribution underneath various substrates soon after instability. (b) Number of drops as a function of drop radius, just before the largest drop leaves the surface. (c) Effect of the choice of the substrate on percentage area of coverage. (d) Variation of heat transfer coefficient with time over various substrates. 
immediately after fall-off when the condensing fluid is sodium $\left(T_{\text {sat }}=377^{\circ} \mathrm{C}, \Delta T_{\text {sat }}=5^{\circ} \mathrm{C}\right.$, $\left.\theta_{\text {avg }}=108^{\circ}\right)$ compared to water $\left(T_{\text {sat }}=27^{\circ} \mathrm{C}, \Delta T_{\text {sat }}=5^{\circ} \mathrm{C}, \theta_{\text {avg }}=95^{\circ}\right)$. For liquid sodium, the drop size at fall-off $\left(r_{\max }\right)$ and the corresponding time from fresh nucleation are $6.7 \mathrm{~mm}$ and $20 \mathrm{~min}$, respectively. For water, these parameters are $3.8 \mathrm{~mm}$ and $57 \mathrm{~min}$. Thus, it can be concluded that smaller drops will form during condensation of sodium vapor, on an average, when compared to water. The cycle time for water is also longer, indicating weaker unsteadiness. Between water and liquid sodium, the heat transfer coefficient would be greater for the latter and would also be accompanied by a larger shear stress.

The number of nucleation sites is chosen as a parameter for condensation underneath a horizontal substrate, the working fluid being water. Nucleation site densities chosen are $10^{4}, 10^{5}$, $10^{6}$ and $10^{7} / \mathrm{cm}^{2}$ respectively, at various degree of subcooling, while the saturation temperature is $27^{\circ} \mathrm{C}$, figure 17 . The overall heat transfer coefficient exhibits a clear positive correlation with the nucleation site density. As the number of nucleation sites increases per unit area, many small drops nucleate on exposure of the surface to vapor, i.e., the average drop size within a cycle decreases. The conduction resistance is thus lowered, leading to an increase in average heat transfer coefficient.

The following discussion simulates condensation of water vapor on a substrate with nucleation site density equal to $10^{6} \mathrm{~cm}^{-2}$. The substrates considered are (i) horizontal, with a wettability gradient - called a graded surface, (ii) horizontal, with uniform wettability, and, (iii) an inclined surface $\left(5^{\circ}\right.$ with horizontal). Condensation of water vapor occurs on the underside of the

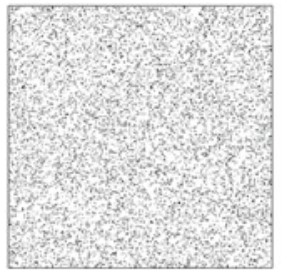
$\mathrm{t}=1 \mathrm{~min}$
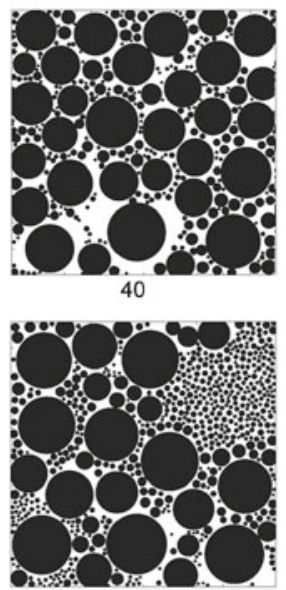

65

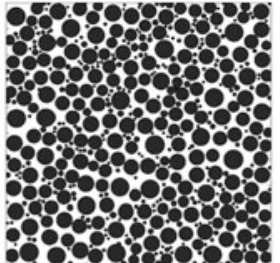

10

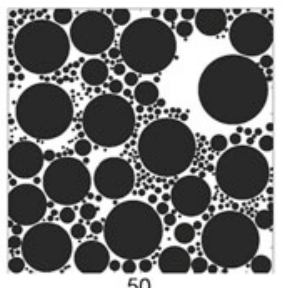

50

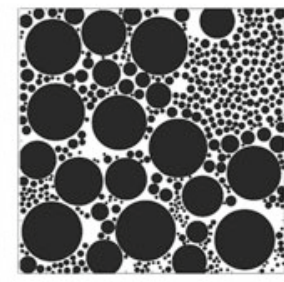

70

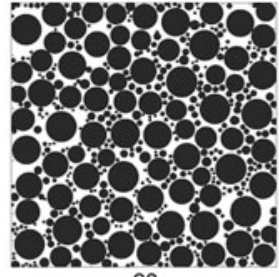

20

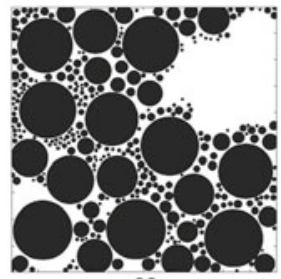

60

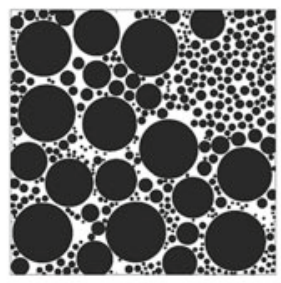

72

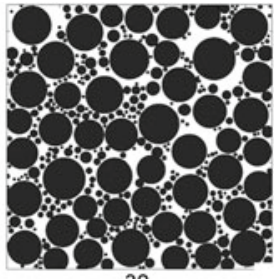

30

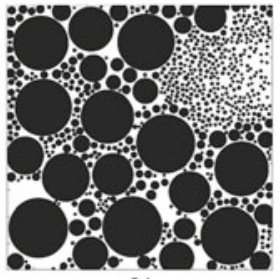

61

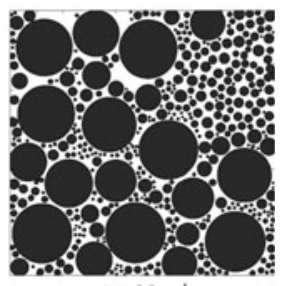

$\mathrm{t}=80 \mathrm{~min}$.

Figure 19. Spatio-temporal distribution of drops formed during dropwise condensation of water vapor at $27^{\circ} \mathrm{C}$ with sub-cooling of $\Delta T=5^{\circ} \mathrm{C}$ underneath a horizontal substrate of size, $20 \mathrm{~mm} \times 20 \mathrm{~mm}$ and apparent contact angle is $95^{\circ}$. 
substrate and the simulation is carried out over an area of $20 \mathrm{~mm} \times 20 \mathrm{~mm}$. Accordingly, liquid drops formed are in the pendant mode at all times. Specifically, the degree of subcooling is $\Delta T_{\text {sat }}=5^{\circ} \mathrm{C}$ with a saturation temperature of $27^{\circ} \mathrm{C}$. Simulation shows that the mathematical model captures the underlying mechanism of dropwise condensation over each of these surfaces. The features of the condensation cycle underneath a horizontal substrate with wettability gradient are similar to those of an inclined surface shown in figure 1. The points of difference are: (i) drops shift towards the lower wettability side, (ii) for a graded surface, drops of all sizes are in motion, and (iii) larger drops acquire greater terminal velocity. Spatial distribution of drops at an instant just before the first drop leaves the surface on a graded substrate, first slide-off from an inclined substrate and the first fall-off from a horizontal substrate, respectively, are compared in figure 18a. As wettability gradient induces motion to drops of every size, there exists an exposed virgin area behind every droplet on the graded substrate, as shown in figure 18a. Hence, the fraction of total area exposed for fresh condensation tends to be greater for a graded surface as compared to the other two configurations. Figure $18 \mathrm{~b}$ shows the frequency of occurrence of a drop of a given radius, namely the histogram, on the three substrates, at an instant just before the slide-off or fall-off criticality is attained. Drops slide-off for a graded surface as well as for the inclined one. Drops fall-off on a horizontal surface. From figure 18b, it is clear that a graded substrate has larger number of smaller sized drops as compared to the other two. Largest drops are formed on a horizontal substrate before they fall-off. This eventually leads to a slower condensation rate on the horizontal substrate; in this regard the graded substrate shows a clear promise
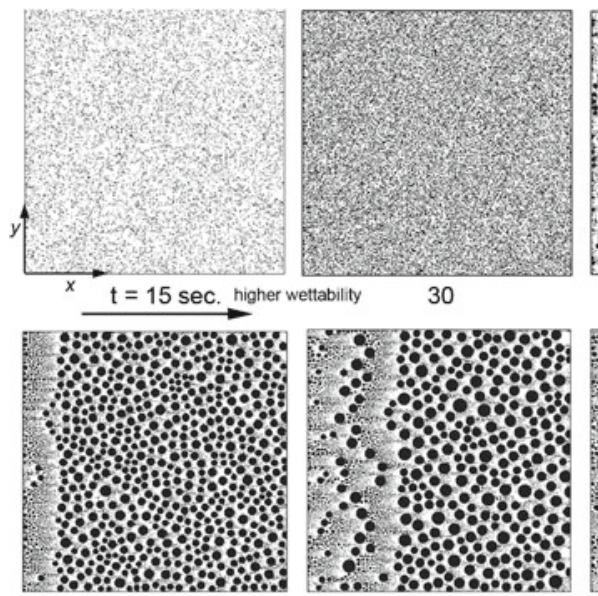

120

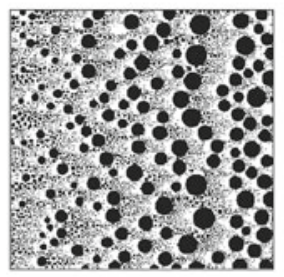

200

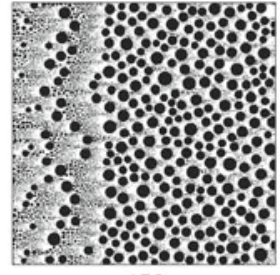

150

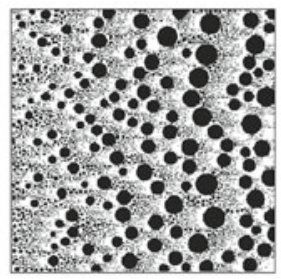

250

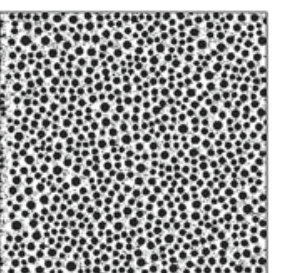

40

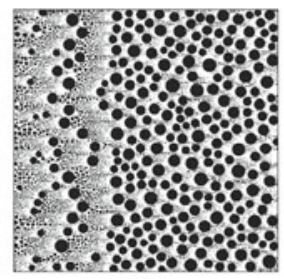

160

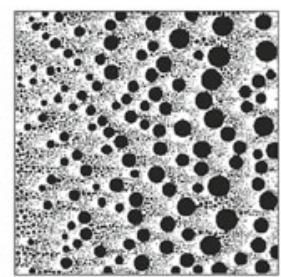

260

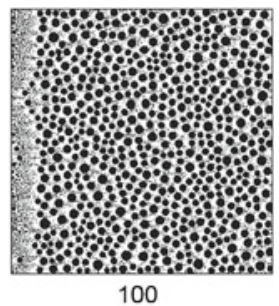

100

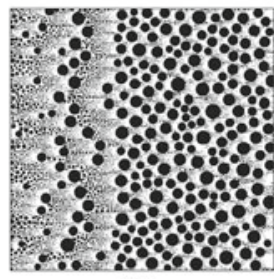

170

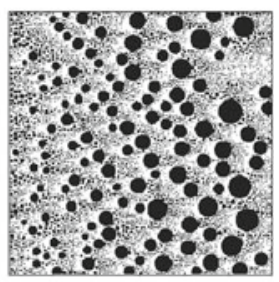

$\mathrm{t}=270 \mathrm{sec}$

Figure 20. Spatio-temporal distribution of drops formed during dropwise condensation of water vapor at $27^{\circ} \mathrm{C}$ with sub-cooling of $\Delta T=5^{\circ} \mathrm{C}$ underneath a horizontal substrate with a wettability gradient of $1.5^{\circ} / \mathrm{mm}$. Maximum contact angle is $110^{\circ}$, minimum contact angle is $80^{\circ}$ and substrate size is $20 \mathrm{~mm} \times 20 \mathrm{~mm}$. 
from a perspective of heat transfer enhancement. Figure 18c shows the area of coverage created by the foot-prints of the drops on the substrate, as a function of time. As soon as the virgin substrate is exposed to vapor flux, direct condensation is initiated and the area coverage of drops increases rapidly. Later, coalescence dominates direct condensation eventually leading to droplet criticality. The cycle is then established and the area coverage tends to stabilize. On a horizontal substrate, only a fall-off criticality is possible while on a graded substrate, a slide-off criticality is usually achieved first. During sliding motion, a droplet may fall-off in transit due to increase in its weight. A quasi-steady-state is eventually reached, after which the area coverage oscillates around an average value.

Results shown in figure 18 reveal that area coverage is smaller for the graded surface, making the exposed area greater than the other two surfaces considered. Further, drop instability in the form of a slide-off event is relatively early on the graded surface. As a direct consequence, heat transfer coefficient is expected to be higher for a surface with variable wettability. Heat transfer rates computed on these surfaces were found to be 450 (horizontal), 520 (inclined) and 540 (graded horizontal) in units of $\mathrm{kW} / \mathrm{m}^{2}$, with a sub-cooling of $5^{\circ} \mathrm{C}$ and a condensation temperature of $27^{\circ} \mathrm{C}$.

For a degree of sub-cooling equal to $5^{\circ} \mathrm{C}$ and a condensation temperature of $27^{\circ} \mathrm{C}$, the spatial and temporal distribution of drops for vertical and horizontal substrates, and a horizontal substrate with wettability gradient, are shown in figures 19-21. The condensation pattern of mercury vapor over a vertical substrate is shown for comparison in figure 22. The duration

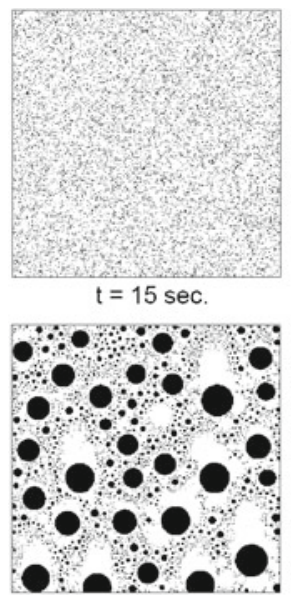

120

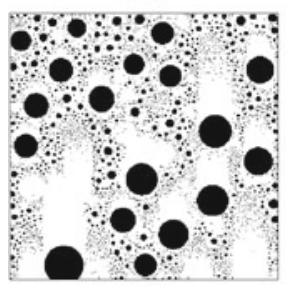

200

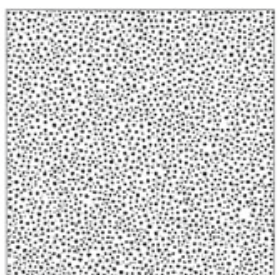

30

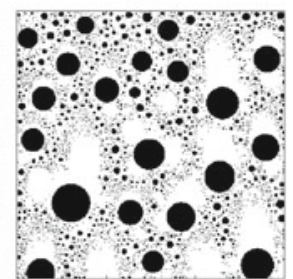

150

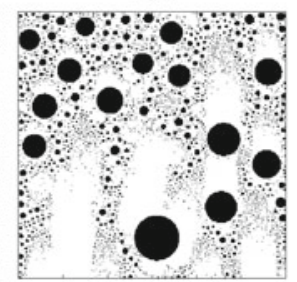

210

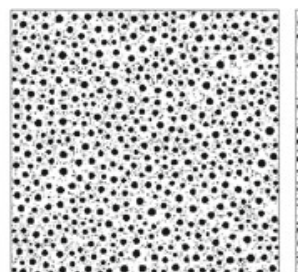

40

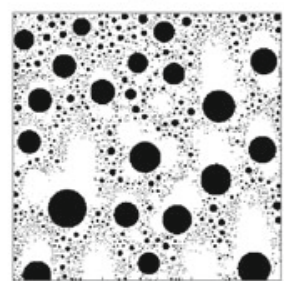

160

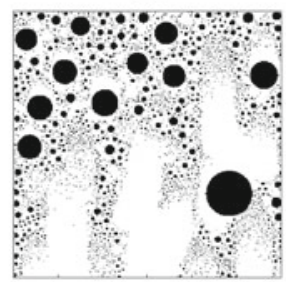

220
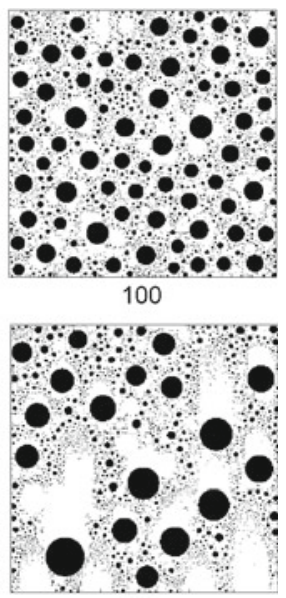

170

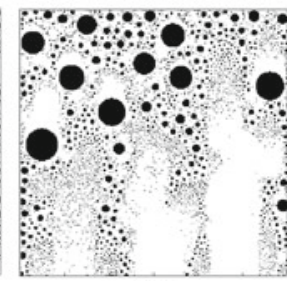

$\mathrm{t}=240 \mathrm{sec}$

Figure 21. Spatio-temporal distribution of drops during dropwise condensation of water vapor at $27^{\circ} \mathrm{C}$ with sub-cooling of $\Delta T=5^{\circ} \mathrm{C}$ along a vertical substrate. The advancing angle is $110^{\circ}$, receding angle is $80^{\circ}$ and substrate size is $20 \mathrm{~mm} \times 20 \mathrm{~mm}$. 

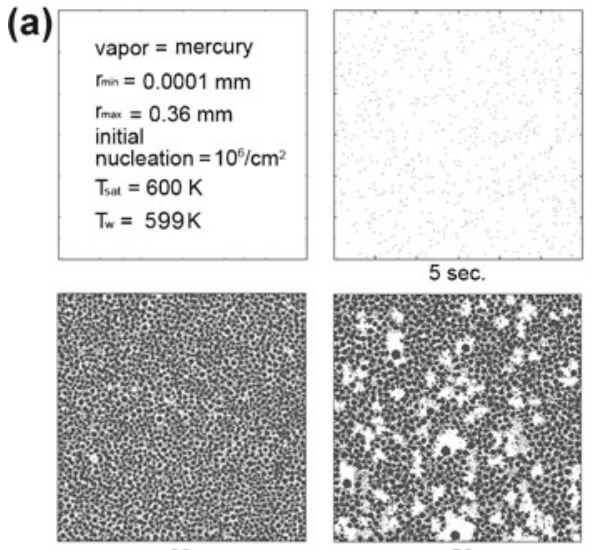

20

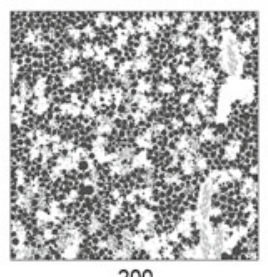

200

(b)
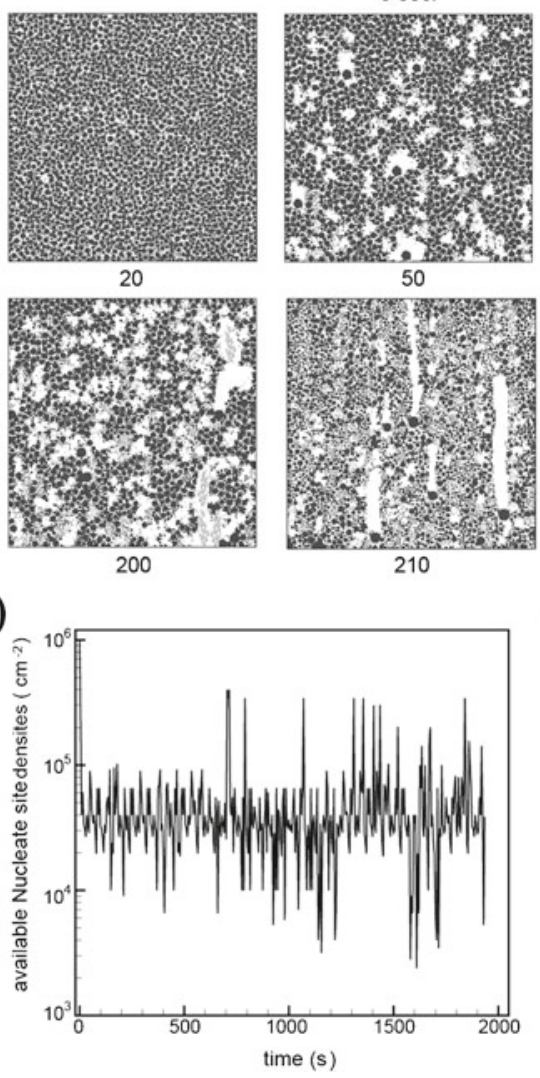

50

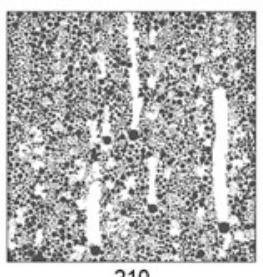

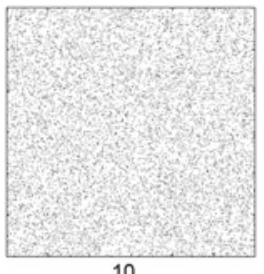
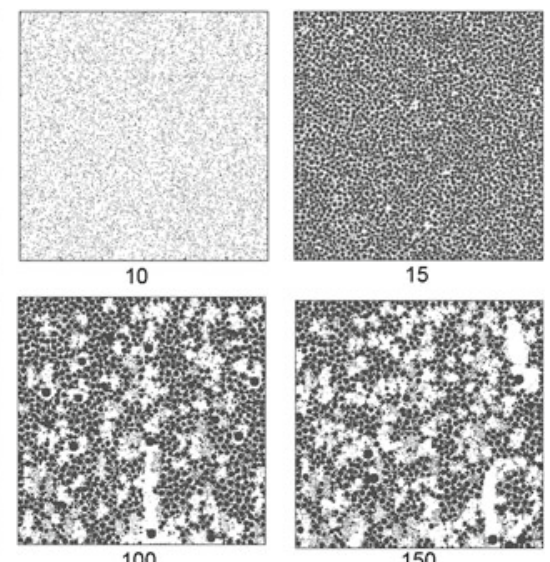

100
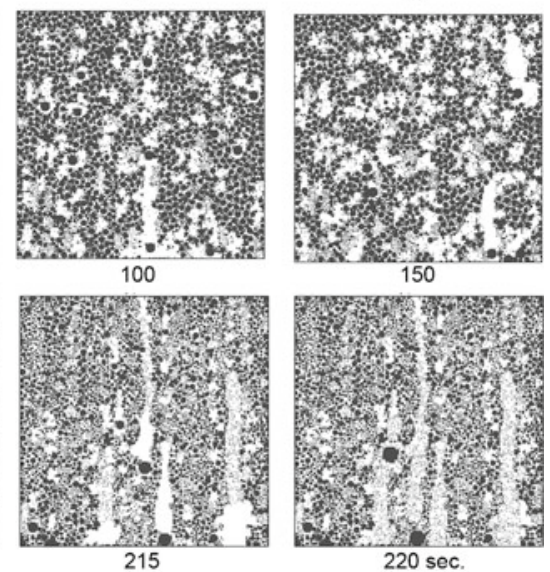

(c)

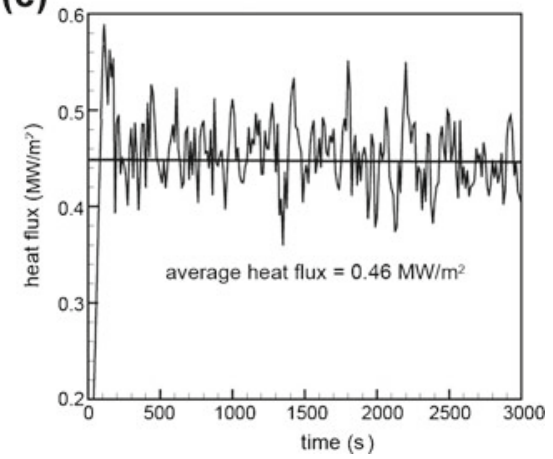

Figure 22. Spatio-temporal distribution of drops during dropwise condensation of mercury at a saturation temperature of $327^{\circ} \mathrm{C}$ with sub-cooling of $\Delta T=30^{\circ} \mathrm{C}$ along a vertical substrate. The advancing angle is $136^{\circ}$, receding angle $124^{\circ}$ and substrate size is $20 \mathrm{~mm} \times 20 \mathrm{~mm}$.

considered in figure 19 is from initial nucleation to the first fall-off underneath a horizontal surface. Figure 20 shows condensation patterns below a horizontal substrate with wettability gradient, beginning from initial nucleation to till a dynamic steady-state is reached. This approach is also followed for a vertical substrate in figure 21 . The similarity in patterns between a vertical substrate (figure 21) and a horizontal graded surface (figure 20) is noticeable. The time cycle from initial nucleation to the instant when the drop leaves the surface is a minimum for the substrate with a wettability gradient. For such graded surfaces, it is observed that smaller drops move with small velocity and larger drops with larger velocity. For an inclined substrate, only the drop that reaches the critical size is set in motion. For the horizontal surface, there is no sliding motion possible; the drop falls off when it achieves a critical size. For the surface with 
wettability gradient, both slide off and fall-off are possible, the latter scenario arising from the accumulated weight of the sliding drop exceeding the retention surface force. The mathematical model developed clearly distinguishes these test cases and is hence of considerable generality.

\section{Conclusions}

A mathematical model of dropwise condensation on textured surfaces, horizontal and inclined, is reported in the present study. The model starts from the smallest thermodynamically stable drop at randomized nucleation sites and follows its growth by direct condensation and coalescence till the drop is large enough to fall-off or slide away. It can also accept an atomistic model as a starting point with the largest atomic cluster serving as the smallest drop at the continuum scale. The falling criterion along with the sliding are developed on the basis of a force balance equation at the level of a drop. The mathematical model accepts thermophysical properties of vapor and liquid phases, those of the substrate, surface tension, and contact angles, as input. Correlations from a CFD model describe wall shear stress and heat fluxes when the drop moves over the surface. The overall model is found to be general enough to describe condensation of water vapor on one hand, all the way to liquid metals such as mercury and sodium on the other. Specific conclusions arrived at in the present study are listed below.

(i) The model shows that dropwise condensation is necessarily a quasi-cyclic process.

(ii) The average drop size, distribution, and cycle time as well as the overall heat transfer coefficient and wall shear can be computed from model simulation.

(iii) Liquid sodium shows drops of bigger average diameter but a smaller cycle time compared to water, and consequently experiences a higher average heat transfer coefficient.

(iv) Providing wettability gradient serves the purpose of passively destabilizing drops in a manner similar to inclined surfaces in gravity fields. It has the advantage of being functional in micro-gravity situations.

(v) With a graded surface, drops of various sizes are in a state of motion, while, for an inclined surface, the largest drop alone is set in motion.

(vi) With the number of parameters available, surface texturing can be seen as a route towards controlling dropwise condensation in engineering applications.

(vii) The heat transfer coefficient of condensing vapor strongly depends on the substrate orientation and hydrophobicity of the substrate. As a rule, an inclined surface will have a higher heat transfer coefficient compared to the horizontal, becoming maximum for a vertical surface.

\section{Acknowledgements}

The authors are grateful to the Board of Research in Nuclear Sciences (BRNS), Department of Atomic Energy, Government of India, for financial assistance. Technical discussions with Dr. L M Gantayet and Dr. Jaya Mukherjee of Bhabha Atomic Research Centre, Mumbai, India, are gratefully acknowledged.

\section{Nomenclature}

A Surface area, $\mathrm{m}^{2}$

$C_{p} \quad$ Specific heat at constant pressure, $\mathrm{W} / \mathrm{kg}-\mathrm{K}$ 
$d_{b} \quad$ Base diameter of drop, $\mathrm{m}$

F Force, N

$h_{l v} \quad$ Latent heat of vaporization, $\mathrm{J} / \mathrm{kg}$

$h \quad$ Heat transfer coefficient, $\mathrm{W} / \mathrm{m}^{2}-\mathrm{K}$

$k \quad$ Thermal conductivity of condensate, $\mathrm{W} / \mathrm{m}-\mathrm{K}$

$N \quad$ Number of nucleation sites, per $\mathrm{cm}^{2}$

$p \quad$ Pressure, $\mathrm{N} / \mathrm{m}^{2}$

$q \quad$ Surface heat transfer, $\mathrm{W}$

$q^{\prime \prime} \quad$ Average heat flux, $\mathrm{W} / \mathrm{m}^{2}$

$r \quad$ Radius of drop, $\mathrm{m}$

$l \quad$ Distance between two nucleation sites, $\mathrm{m}$

$T \quad$ Temperature, $\mathrm{K}$

$\Delta T \quad$ Temperature difference between the saturated vapor and condensing wall, $\mathrm{K}$

$\Delta t \quad$ Time step, s

$u, v, w \quad$ Velocity component in $x, y$ and $z$ directions, $\mathrm{m} / \mathrm{s}$

$U \quad$ Relative velocity between the condensing wall and the drop, $\mathrm{m} / \mathrm{s}$

$V \quad$ Volume of the drop, $\mathrm{m}^{3}$

\section{Dimensionless quantities}

$\mathrm{C}_{f}, \overline{\mathrm{C}}_{f}$ Local and average skin friction coefficient, $2 \tau_{w} / \rho U^{2}$

$\mathrm{Nu}, \overline{\mathrm{Nu}}$ Local and average Nusselt Number, $h d_{b} / k_{c}$

Pr Prandtl Number, $\mu C_{p} / k_{c}$

Re Reynolds number, $\rho U d_{b} / \mu$

\section{Greek symbols}

$\alpha \quad$ Inclination angle from horizontal, radian/degree

$\delta \quad$ Thickness of promoter layer, $\mathrm{mm}$

$\mu \quad$ Dynamic viscosity, Pa-s

$v \quad$ Specific volume, $\mathrm{m}^{3} / \mathrm{kg}$

$\rho \quad$ Density, $\mathrm{kg} / \mathrm{m}^{3}$

$\sigma \quad$ Surface tension of liquid, $\mathrm{N} / \mathrm{m}$

$\tau_{w}, \bar{\tau}_{w}$ Local and average wall shear stress, $\mathrm{N} / \mathrm{m}^{2}$

$\theta \quad$ Contact angle, radians or degrees

$\Delta \theta \quad$ Difference in advancing and receding angle, radians or degrees

\section{Subscripts}

$\begin{array}{ll}a d v & \text { Advancing } \\ a v g & \text { Average } \\ b & \text { Base } \\ \text { coat } & \text { Drop promoter coating } \\ \text { cond } & \text { Conduction } \\ \text { const } & \text { Constriction } \\ \text { crit } & \text { Critical }\end{array}$




$\begin{array}{ll}\text { int } & \text { Interface } \\ l & \text { Liquid } \\ l v & \text { Liquid-vapor interface } \\ r c d & \text { Receding } \\ \text { max } & \text { Maximum } \\ \text { min } & \text { Minimum } \\ \text { sat } & \text { Saturation } \\ s l & \text { Solid-liquid interface } \\ \mathrm{v} & \text { Vapor } \\ \mathrm{w} & \text { Wall }\end{array}$

\section{References}

Amar J G, Popescu M N and Family F 1999 Self-consistent rate equation approach to nucleation and growth in point/extended island models of 1-D homoepitaxy. Proc. Mater. Res. Soc. 570(3): 1-9

Bansal G D, Khandekar S and Muralidhar K 2009 Measurement of heat transfer during dropwise condensation of water on polyethylene. Nanoscale and Microscale Thermophys. Eng. 13(3): 184-201

Barth T J and Jespersen D C 1989 The design and application of upwind schemes on unstructured meshes. AIAA 89: 0366

Berthier J 2008 Microdrops and digital microfluidics (second ed.). USA: Hemisphere Publishing Corporation, pp. 75-179

Brennen P J and Kroliczek E J 1979 Heat pipe design handbook, NASA Report NAS5-23406

Brune H 1998 Microscopic view of epitaxial metal growth: Nucleation and aggregation. Surf. Sci. Rep. 31(3): 121-229

Carey V P 1992 Liquid-vapor phase-change phenomena (second ed.). USA: Hemisphere Publishing Corporation, pp. 413-472

Daniel S, Chaudhury M K and Chen J C 2001 Fast drop movements resulting from the phase-change on a gradient surface. Science 291: 633-636

Date A W 2005 Solution of transport equations on unstructured meshes with cell centered collocated variables. Part I: Discretization. Int. J. Heat Mass Transf. 48: 1117-1127

Elsherbine A I and Jacobi A M 2006 Retention forces and contact angles for critical liquid drops on nonhorizontal surfaces. J. Colloid Interf. Sci. 299: 841-849

Frink N T, Paresh P and Shahyar P 1991 A fast upwind solver for the Euler equations on three-dimensional unstructured meshes. AIAA 91: 0102

Kim S and Kim K J 2011 Dropwise condensation suitable for superhydrophobic surfaces. J. Heat Transf. 133(8): 081502(1-8)

Lan Z, Ma X Z, Zhang Y and Zhou X D 2009 Theoretical study of dropwise condensation heat transfer: Effect of the liquid-solid surface free energy difference. J. Enhanc. Heat Transf. 16: 61-71

Leach R N, Stevens F, Langford S C and Dickinson J T 2006 Dropwise condensation: experiments and simulations of nucleation and growth of water drops in a cooling System. Langmuir 22: 8864-8872

Leipertz A 2010 Dropwise condensation: Eds. P. Stephan: VDI Heat Atlas VDI-GVC (second ed.). Germany: Springer-Verlag, pp. 933-937

Ma X, Wang S, Lan Z, Peng B, Ma H B and Cheng P 2012 Wetting mode evolution of steam dropwise on condensation superhydrophobic surface in the presence of non-condensable Gas. ASME J. Heat Transf. 134: 021501-021509

Mikic B B 1969 On the mechanism of dropwise condensation. Int. J. Heat Mass Transf. 12: 1311-1315

Miljkovic N, Enright R and Wang E N 2012 Effect of droplet morphology on growth dynamics and heat transfer during condensation on superhydrophobic nanostructured surfaces. ACS Nano 6: 1776-1785

Moumen N, Subramanian R and McLaughlin J B 2006 Experiments on the motion of drops on a horizontal solid surface due to wettability gradient. Langmuir 22: 2682-2690 
Mu C, Pang J and Liu T 2008 Effect of surface topography of material on nucleation site density of dropwise condensation. Chem. Eng. Sci. 63: 874-880

Necmi S and Rose J W 1977 Heat-transfer measurements during dropwise condensation of mercury. Int. J. Heat Mass Transf. 20: 877-880

Oura K, Lifshits V G, Saranin A A, Zotov A V and Katayama M 2003 Surface science (first ed.). Springer, pp. 220-260

Pratap V, Moumen N and Subramanian R 2008 Thermocapillary motion of a liquid drop on a horizontal solid surface. Langmuir 24: 5185-5193

Qiang L, Hong W, Xun Z and Mingwei Z 2006 Droplet movement on horizontal surfaces with gradient surface energy. Sci. China, Series E: Technol. Sci. 49: 733-744

Rose J W 1976 Further aspects of dropwise condensation theory. Int. J. Heat Mass Transf. 19: 1363-1370

Rose J W 2002 Dropwise condensation: theory and experiments: A review. Proc. IME 216: 115-118

Rose J, Utaka Y and Tanasawa I 1999 Dropwise condensation: in Handbook of Phase Change: Boling and Condensation/ S G Kandlikar (ed.), USA: Taylor and Francis, pp. 581-594

Rykaczewski K 2012 Microdroplet growth mechanism during water condensation on superhydrophobic surfaces. Langmuir 28: 7720-7729

Shi F, Shim Y and Amar J G 2005 Island-size distribution and capture numbers in three-dimensional nucleation: Comparison with mean-field behavior. Phys. Rev. B 71: 245411-245416

Sikarwar B S, Battoo N K, Khandekar S and Muralidhar K 2011a Dropwise condensation underneath chemically textured surfaces: simulation and experiments. ASME J. Heat Transf. 133(2): 021501 (1-15)

Sikarwar B S, Khandekar S and Muralidhar K 2011b Dropwise condensation on horizontal substrates with and without a wettability gradient. In: $8^{\text {th }}$ Minsk International Seminar Heat Pipes, Heat Pumps, Refrigerators, Power Sources, Minsk, Belarus

Sikarwar B S, Khandekar S and Muralidhar K 2011c Dropwise condensation of liquid metal vapor underneath a flat inclined substrate. In: Proceeding $7^{\text {th }}$ International Conference "Computational heat and mass transfer" Istanbul, Turkey (Paper \#135)

Sikarwar B S, Muralidhar K and Khandekar S 2011d Simulation of dropwise condensation underneath a chemically textured substrate with a wettability gradient. In: Proceeding $21^{\text {st }}$ National and $10^{\text {th }}$ International ISHMT-ASME heat and mass transfer conference, Chennai, India (Paper \# IND_16_035)

Sikarwar B S, Khandekar S, Agrawal S, Kumar S and Muralidhar K 2012 Dropwise condensation studies on multiple scales. Heat Transf. Eng. 33(4-5): 301-341

Sikarwar B S, Khandekar S and Muralidhar K 2013 Simulation of flow and heat transfer in a drop sliding underneath a hydrophobic surface. Int. J. Heat Mass Transf. 57: 786-811

Subramanian R, Moumen N and McLaughlin J B 2005 The motion of a drop on a solid surface due to a wettability gradient. Langmuir 21: 11844-11849

Tien C L, Majumdar A and Gerner F M 1997 Microscale energy transport. Series in Chemical and Mechanical Engineering, USA: Taylor and Francis, pp. 165-185

Tian Y, Wang X D and Peng X F 2004 Analysis of surface inside metastable bulk phase during gas-liquid phase transition. J. Eng. Thermophys. (in Chinese) 25: 100-102

Venables J A 2000 Introduction to surface and thin film processes (first ed.). Cambridge, UK: Cambridge University Press, pp. 144-165

Venables J A and Brune H 2002 Capture numbers in the presence of repulsive adsorbate interactions. Phys. Rev. B 66: 195404-195419

Wang X D, Tian Y and Peng X F 2003 Self-aggregation of vapor-liquid phase transition. Progress Nat Sci. (Chinese) 13: 281-286

Zhao H and Beysens D 1996 From droplet growth to film growth on a heterogeneous surface: condensation associated with a wettability gradient. Langmuir 11: 627-634 